\title{
Organización de la información mediante el uso de lenguajes de modelado: viejos recursos para nuevas necesidades
}

\author{
Alejandro Delgado Gómez \\ Ayuntamiento de Cartagena
}

\subsection{Resumen}

Primero, se establece el contexto en el que nace la necesidad de elaborar una ontología. Después, se discuten los conceptos de modelo y de ontología, y se justifica la selección de un concepto intensional. Se discute de igual modo la diferencia entre el diseño de ontologías y la reingeniería de procesos. Finalmente, se explican con más detalle el método y los lenguajes IDEF5 para la creación de ontologías. Se refieren algunos ejemplos de software relevante.

Palabras clave: Ontologías. Lenguajes de modelado. IDEF5.

\subsection{Abstract}

First, the context in which the need to develop an ontology appears is defined. Then, we discuss the concepts of "model" and "ontology", and justify our bet for an intensional concept. In the same way, we discuss the differences between ontologies design and business processes re-engineering. Finally, the IDEF5 method and languages to create ontologies are explained in a more detailed way, refering to some examples of associated software.

Keywords: Ontologies. Modelling languages. IDEF5.

\section{Contextualización del problema}

Ante todo, debe precisarse que el estudio cuyo desarrollo a continuación se esboza nace de una necesidad puramente práctica, la de reorganizar la información de una institución grande y compleja. En este caso, además, puede considerarse dicha información como multidisciplinar o, de manera más débil, procedente de diversas áreas que, por otra parte, mantienen relaciones igualmente complejas y no lineales. Además, estas áreas han generado su información incontroladamente y sin procurar la debida consistencia ni interna ni externa. Es decir, el problema consiste en reorganizar una ingente cantidad de información que se encuentra cercana al caos y que crece continuamente. Por ello, no resulta de uti- 
lidad un lenguaje tradicional de análisis, como por ejemplo simples diagramas de flujo, que reflejan una realidad secuencial. Pero, habida cuenta de que en el escenario arriba esbozado no sólo existe información, sino también, entre otras cosas, personas que la generan, tampoco resulta de utilidad un lenguaje altamente abstracto, como por ejemplo KIF (Knowledge Interchange Format) (Geneserethy Fikes, 1992), legible por el ser humano pero ideado para servir de lenguaje de intercambio entre ordenadores. Por el mismo motivo no podemos utilizar proyectos creados para su uso en contextos automatizados, por ejemplo OILEd (1) o SHOE (2), ni aquellos que, aun siendo inteligentes y amigables, se concentran en un solo tipo de relación, por ejemplo Protégé (3). Obviamente, no podemos pensar en ninguna técnica de reingeniería de procesos, puesto que nuestra función no es poner orden en la institución misma, sino en la información que genera. Así, pues, precisamos de una herramienta que: 1) sea utilizable por usuarios con distinto nivel de destreza y permita el diálogo con personas que no son analistas de información; 2) sea utilizable de manera manual, y esto significa incluso que el proyecto pueda quedar terminado haciendo uso simplemente de papel y lápiz, aunque pueda trasladarse a un entorno automatizado; 3) sea lo suficientemente exhaustiva como para analizar y definir la información, sus componentes y relaciones de la manera más precisa posible y con independencia de las instancias con las que nos encontramos, a fin de evitar que el análisis sea un espejo del caos de estas instancias; 4) sea lo suficientemente sólida como para permitir la durabilidad de la reorganización de la información, una vez finalizado el proyecto.

En lo que sigue se explica el estudio previo a la selección de esta herramienta y las características de la herramienta seleccionada. Debe tenerse en cuenta, insistimos, el hecho de que incluso cuando nos dejamos llevar por vericuetos teóricos, estamos pensando en el fin práctico ya esbozado. Estamos de acuerdo con Gruber en la afirmación de que una ontología es siempre una ontología "para algo" (4).

Por último, debemos informar de que lo que se describe a continuación es la historia de un fracaso: creemos que tanto el estudio para la selección de esta herramienta como la herramienta seleccionada son correctos y funcionan. La responsabilidad del fracaso corresponde al analista, quien olvida que una premisa fundamental a la hora de gestionar información es incluir, como ya se adelantó y se tratará más adelante, a las personas entre los objetos que forman parte del dominio, y que ningún proyecto de organización tendrá éxito si estas personas que forman parte del dominio se resisten a ello.

\section{Lenguajes de modelado y ontologías}

Consideramos tres tipos de procedimiento, expuestos a continuación en orden ascendente en cuanto a su capacidad de abstracción: 
1) Modelo: IDEF0 define un modelo como "una representación de una serie de componentes de un sistema o un área temática" (5). En líneas generales, un modelo es un procedimiento de comprensión y análisis de un sistema para su mejora o substitución. Aunque lo que se trata de analizar, en sentido amplio, es un sistema, en nuestro caso éste se compone de múltiples áreas temáticas relacionadas de manera inconsistente. La utilización de modelos supone el riesgo de convertirse en un espejo del caos que intentamos solventar. No podemos, por tanto, aceptar este procedimiento, sino, más bien, buscar otro que proponga un nivel de abstracción mayor.

2) Ontología (en acepción extensional): La definición más extendida de ontología es quizá la de Tom Gruber: "una ontología es una especificación de una conceptualización" (6), queriendo significar con ello la descripción de conceptos y relaciones que pueden existir para un agente dado o una comunidad de agentes. La definición de ontología de Gruber es extremadamente práctica y bien pudiera servir a nuestro propósito; pero depende de las instancias de las relaciones en un dominio dado, riesgo que, como adelantamos, tratamos de evitar. Así, pues, a esta definición se le puede contra-argumentar, como así hace Nicola Guarino (1998a), el hecho de que está basada en una noción extensional de las relaciones, mientras que éstas debieran ser independientes de sus instancias, es decir, intensionales. Así, el argumento de Guarino refina la versión de Gruber.

3) Ontología (en acepción intensional): Por una parte, para Guarino una ontología se diferencia de un modelo en al menos dos peculiaridades: una de ellas metodológica y la otra arquitectónica. Así, en primer lugar, una ontología adopta una aproximación altamente interdisciplinar; y, en segundo, juega un papel central en un sistema de información, de tal manera que bien pudiera llegar a hablarse de sistemas de información orientados por ontología. Por otra parte, Guarino formaliza la definición de ontología en términos de lógica formal para llegar a la siguiente definición:

una ontología es una 1) teoría lógica que 2) da cuenta del significado pretendido de un vocabulario formal, es decir, 3) su compromiso ontológico con una 4) determinada conceptualización del mundo. Los modelos pretendidos de un lenguaje lógico que usa tal vocabulario están restringidos por su compromiso ontológico. Una ontología refleja indirectamente este compromiso (y la conceptualización subyacente), aproximándose a estos modelos pretendidos.

Como se puede apreciar, en esta definición aparece un cierto número de conceptos, la formalización de cada uno de los cuales es dependiente de la de los demás. La argumentación de Guarino sigue el camino inverso al que se muestra en la definición, y la explicamos en filigrana, a efectos de clarificación, aunque tratamos de evitar en lo posible el lenguaje formal.

Scire. $8: 1$ (en.-jun. 2002) 55-86. 
En términos generales, cuando se habla de ontología se piensa en dos acepciones: la filosófica, es decir, un determinado sistema de categorías que dan cuenta de una determinada visión del mundo; y la que prevalece en el contexto de la inteligencia artificial, es decir, un 1) producto de ingeniería 2) constituído por un vocabulario específico 3) utilizado para describir una cierta realidad, más una 4) serie de asumpciones explícitas relativas al significado pretendido de las palabras del vocabulario. Dicha serie de asunciones se muestra como una teoría lógica de primer orden, donde las palabras del vocabulario son términos simples o binarios, llamados respectivamente conceptos y relaciones. Es esta acepción la que utilizaremos en lo sucesivo para hablar de ontología, reservando el término "conceptualización" para la primera acepción. A simple vista, ya percibimos que una ontología es de naturaleza lingüística, mientras que una conceptualización no lo es.

Como dijimos, el argumento de Guarino parte del concepto de intensionalidad, y una manera razonablemente aceptada de definir intensiones consiste en considerarlas como funciones de mundos posibles dentro de series; pero, mientras que las relaciones ordinarias se aplican sobre dominios o estados de cosas dados, las relaciones conceptuales o intensionales se aplican sobre lo que Guarino llama "espacios de dominio". Es decir, una estructura de Gruber, para expresar una conceptualización, tiene la forma "dominio, relaciones en el dominio", que sólo es aplicable a un mundo, mientras que una estructura de Guarino para el mismo fin tiene la forma "dominio, conjunto de mundos posibles en ese dominio, conjunto de relaciones admisibles dentro de cada uno de esos mundos". La serie de relaciones, en la estructura de Guarino, ya no es extensional, sino ella misma intensional, e incluye todas las extensiones admisibles de una relación de exponente n, entendida como una función total a partir del conjunto de mundos posibles en la serie de todas las relaciones ordinarias n-arias en el dominio en cuestión. Una conceptualización contiene una de tales estructuras para cada mundo posible. En la terminología de Guarino, estas estructuras se llaman estructuras pretendidas de mundo.

Hasta el momento ha quedado definida la noción de conceptualización que subyace a una ontología; pero, como dijimos, una ontología es de naturaleza lingüística, o, en sentido amplio, refleja lingüísticamente una conceptualización. Por ello debemos tomar ahora un determinado lenguaje lógico, con un vocabulario definido. Según el argumento de Guarino, una expresión para ese lenguaje lógico es una estructura de la forma "estructura de mundo, función de interpretación que asigna elementos del dominio a símbolos constantes del vocabulario y elementos de un conjunto de relaciones a símbolos de predicado del vocabulario". La anterior es la versión extensional del lenguaje; en su versión intensional, utilizamos la estructura "conceptualización, función que asigna elementos del dominio a símbolos constantes del vocabulario y elementos de la serie de extensiones admisibles de una relación a símbolos de predicado del vocabulario". Esta interpretación intensional

Scire. $8: 1$ (en.-jun. 2002) 55-86. 
se llama "compromiso ontológico" del lenguaje lógico dado, lo cual viene a significar que si esta estructura es el compromiso ontológico de dicho lenguaje lógico, entonces este lenguaje se compromete con la conceptualización dada en la estructura por medio de tal compromiso ontológico, mientras que aquella conceptualización subyace a este compromiso ontológico. La serie de todos los modelos del lenguaje lógico que son compatibles con su compromiso ontológico se llama la serie de modelos pretendidos del lenguaje lógico según su compromiso ontológico.

Mediante la precedente explicación, Guarino llega a la siguiente definición amplia de ontología: "Dado un lenguaje L con un compromiso ontológico K, una ontología para L es una serie de axiomas diseñados de tal modo que la serie de sus modelos se aproxima tanto como es posible a la serie de modelos pretendidos de $\mathrm{L}$ según K". Como se ve, esta argumentación refina notablemente la de Gruber y permite un análisis más detallado de diferentes ontologías, para verificar su validez según los propósitos del analista. A pesar de ello, deben hacerse dos anotaciones a la definición de ontología de Guarino. La primera de ellas, de la que es consciente y declara haciendo uso de la expresión "tanto como es posible", es que toda ontología tiene un margen de indeterminación y nunca es completa. En la transición conceptualización-lenguaje-ontología se sacrifica la exhaustividad en aras de la precisión. La segunda anotación es nuestra y constituye más bien una queja: en el contexto de la inteligencia artificial, resulta difícil encontrar una definición bien establecida de "axioma", similar a la definición bien establecida por la filosofía. La definición más precisa, fuerte y aproximada a la bien establecida definición filosófica es la que maneja el proyecto Ontolingua: "Un axioma es una sentencia de lógica de primer orden de la que se asume que es verdadera sin prueba. En la práctica, utilizamos axiomas para referirnos a sentencias que no pueden representarse usando sólo slots y valores de una estructura" (9). Provisionalmente, definimos axioma tal y como lo hace IDEF5, método al que dedicamos la mayor parte de este trabajo: "un axioma es una caracterización precisa de la lógica de un término o serie de términos relacionados, declarados en el lenguaje de elaboración de IDEF5. Un axioma expresa típicamente una restricción sobre los objetos denotados por los términos del axioma" (8). Como puede intuirse, la intención diríase similar a la de un "axioma filosófico", pero su expresión resulta mucho más ambigua y débil, en cualquiera de las dos definiciones. No obstante, ya decimos que renunciamos, en beneficio de la coherencia, a substituir las definiciones autorizadas por los lenguajes en uso por una definición filosófica fuerte. Por otra parte, una definición fuerte podría acrecentar el riesgo de anular la utilidad del lenguaje en un dominio, digamos, "real".

\section{Diferentes ontologías}

Como se adelantó en la introducción, existen, en efecto, muchas ontologías, con muchos proyectos asociados y diferentes grados de normalización. A pesar de

Scire. $8: 1$ (en.-jun. 2002) 55-86. 
la aproximación interdisciplinar de la que hablábamos, algunas se han desarrollado para satisfacer un fin específico en un determinado contexto, pareciéndose más a un lenguaje de modelado que a una ontología como tal. Otras, al contrario, poseen un grado de abstracción tal que las hace ineficientes a la hora de enfrentarse a un problema concreto. Para referirnos sólo a algunos de los lenguajes mejor aceptados, Knowledge Interchange Format (KIF) no ha sido diseñado para ser utilizado por humanos, aunque un humano lo puede utilizar; Resource Description Framework (RDF) (9), sus esquemas y su pretendida extensión que lo mejora, Ontology Interchange Language (OIL) dependen, por lo que en nuestro contexto y a nuestro fin se refiere, en exceso del concepto informático de lenguaje de metadatos. Algunas realizaciones —Protégé, SHOE, CYC (10)...- son productos terminados y, por supuesto, ideados como aplicación informática, no para uso manual.

Entiéndase en este punto que no estamos cuestionando la validez de un lenguaje o de un producto. Todo aquel que cumpla los requisitos del argumento de Guarino que especificamos más arriba nos parece válido. Estamos hablando simplemente de una cuestión práctica: el lenguaje que nosotros necesitamos en un determinado momento y para ciertos fines debe, no sólo satisfacer los requisitos del argumento de Guarino, sino además ser independiente de la máquina, aunque incorporable a ella, y, lo que es más importante, de utilización rápida y sencilla. Algo más adelante explicamos nuestra decisión, que quizá no sea la más avanzada ni la más adecuada en términos generales, pero sí la que precisamos en el contexto dado al comienzo. Por otra parte, debe quedar claro que el método seleccionado depende profundamente de Knowledge Interchange Format, es decir, de la teoría extensional debida a Genesereth y desarrollada por Gruber, lo cual contradice el principio de intensionalidad de Guarino. Sin embargo, este método pretende, al menos, "intensionalizar" algunos de sus componentes. Puesto que el método, no obstante, cumple con los requisitos prácticos que exigimos, aceptamos esta contradicción interna, y respetamos sus restricciones, aunque no compartimos algunas de ellas. Esperamos que esto quede algo más claro en lo que sigue.

\section{4. ¿Reingeniería de procesos u organización del conocimiento?}

Como se adelantó, la necesidad de aproximarse al diseño de ontologías no es, en nuestro caso, puramente teórica, sino derivada de un problema práctico: la obligación de organizar la información generada por una organización de amplio tamaño, multidisciplinar, inconsistente entre diversas áreas, dotada de relaciones complejas, difusas y no lineales; y en estado casi caótico. Además, el resultado de esta organización debe haber sido también una aplicación informática capaz de evitar el peligro de que, solucionada la situación, ésta llegue a repetirse. Dígase de pasada que esta aplicación informática se creó y se puso parcialmente en uso, aunque el no considerado "factor humano" acabó con ella.

Scire. $8: 1$ (en.-jun. 2002) 55-86. 
Aunque una ontología debe tender a la multidisciplinariedad, sí es cierto que muchas de ellas se concentran en área específicas, pero no, curiosamente, en un área tradicionalmente tan necesitada de organización como la Administración pública. El área de trabajo más similar que encontramos es la de los negocios o la empresa en sentido amplio. Véase, a modo de ejemplo, algunas de las conclusiones informales del International Semantic Web Working Symposium (11). Esto nos obliga a repasar en filigrana, por su proximidad a las técnicas de reorganización, las de reingeniería de procesos. Si bien en los campos de la lógica y de la inteligencia artificial se ha utilizado el concepto de ontología desde hace mucho tiempo, manejamos aquí la hipótesis de trabajo de que su actual relevancia en el ámbito empresarial y, por extensión, en la Administración pública, se debe, al menos parcialmente, al fracaso de las técnicas de Business Processes ReEngineering (BPR), en auge durante los años ochenta y primeros noventa. El desarrollo más notable de estas técnicas viene quizá de la mano del Gobierno de los Estados Unidos y, de manera específica, del Departamento de Defensa y sus normas DoD, en lo que viene al caso la DoD 8020.1-M (Functional Process Improvement), ahora parcialmente obsoleta.

Una consecuencia de ella son las técnicas de Functional Economic Analysis (FEA), consideradas aún de relevancia, como puede comprobarse si se visita el sitio del Electronic College of Process Innovation (12). Atendiendo al FEA Guidebook (13), son tres los principios generales que rigen su metodología. El primero es el enfoque sobre las funciones, es decir, sobre la evaluación de los cambios en los procesos funcionales, no en los sistemas de información. Resulta significativo que en el Guidebook se explique que "este enfoque es necesario para asegurar que las inversiones se seleccionan por los beneficios que proporcionarán a las funciones DoD, y no simplemente por consideraciones tecnológicas". El segundo principio es el de la medición de los atributos clave de los procesos funcionales, tales como costes y resultados. El tercer principio es el diseño de FEA como herramienta de gestión permanente, no como requisito de información puntual. En primer lugar, comprobamos que ninguna de estas funciones compete a nuestro fin; de hecho, explícitamente se rechaza el enfoque sobre los sistemas de información. En segundo lugar, si entramos en el software más conocido y "oficializado" para desarrollar la tecnología FEA, Turbo BPR (14), advertimos de un solo pantallazo que no es sino otra aplicación para llevar a cabo un análisis estratégico de mercado al estilo clásico, es decir, más de lo mismo. En tercer lugar, a la reingeniería de procesos le interesa el resultado económico, cayendo fuera de su área de interés el hecho de que la información es un capital, si bien no tangible. Con todo, no dejamos de considerar que los métodos de diseño de ontologías y los de reingeniería de procesos son implícitamente transversales y que ambos debieran tenerse mutuamente en cuenta.

Scire. $8: 1$ (en.-jun. 2002) 55-86. 
Ahora bien, Mayer y deWitte informan en Delivering Results: Evolving BPR from Art to Engineering (15) del fracaso del 70\% de las acciones de reingeniería de procesos emprendidas. Achacan este fracaso a las siguientes razones: la falta de un argumento empresarial adecuado, la ausencia de metodologías y tecnologías robustas y fiables, una implantación incompleta o inadecuada, o el uso indiscriminado de modelos de conocimiento que sirven en cualquier caso. A partir de estas peticiones de principio, desarrollan un modelo diferente, metodológicamente fundado y tecnológicamente sólido, de un análisis económico estratégico. No se adentran en el territorio de lo que hemos definido como ontología, pero se aproximan a él. Además, introducen como método y herramienta el uso de los lenguajes IDEF, aunque, puesto que están diseñando un modelo para mejorar un proceso empresarial, no una ontología, concentran su esfuerzo en el uso de IDEF0 e IDEF3. Estos, como veremos, cumplen en alto grado los requisitos que al comienzo pedíamos al procedimiento buscado, de manera que en su estudio nos concentramos.

\section{Los métodos IDEF}

Los métodos IDEF - ICAM (Integrated Computer Aided Manufacturing) DEFinition- nacen en los años setenta, también como consecuencia de la investigación de las Fuerzas Aéreas norteamericanas para mejorar la productividad. En la actualidad, la investigación acerca de estos métodos continúa, y siguen siendo utilizados de manera creciente como técnicas de modelado de realidades complejas, aunque han ampliado su campo de actividad al desarrollo de bases de datos relacionales, análisis de procesos o, por lo que nos interesa, diseño de ontologías. Como dijimos, el motivo del interés en los métodos IDEF se basa, parcialmente, en el relativo fracaso de las técnicas BPR empleadas en los años ochenta, en la medida en que éstas resultan demasiado dependientes de teorías clásicas de mercado y análisis estratégico, mientras que los métodos IDEF, implícitamente, se van alejando del concepto de productividad y aproximándose al de organización de la información. Por otra parte, un órgano de reconocido prestigio, como el DLM Forum, asigna a los métodos IDEF la categoría "verde", es decir, la de especificación estable y válida, como puede verificarse en la traducción de José María Fernández Hevia de las DLM-Forum Guidelines (16). Es cierto que esta aprobación, en cierto sentido oficial, se refiere sólo a algunos de los lenguajes; pero cabe pensar que ello se debe al hecho de que los demás no se habían desarrollado aún plenamente en aquel momento. Puesto que todos tienen un "aire de familia", e incluso pueden utilizarse, con restricciones, en combinación, no es ilusorio pensar que una nueva edición de las Guidelines aprobara también los lenguajes entonces no totalmente desarrollados: 0, Function Modelling Method; 1, Information Modelling Method; 1x, Data Modelling Method; 3, Process Description Capture Method; 4, ObjectOriented Design Method; 5, Ontology Description Capture Method; 6, Design 
Rationale Capture Method; 8, Human-System Interaction Design Method; 9, Business Constraint Discovery Method; 14, Network Design Method (17).

Sin embargo, habida cuenta de que los métodos IDEF tienen diferentes funcionalidades, aunque un similar aire de familia, el cuerpo de este trabajo se enfoca sobre el desarrollo de ontologías a partir de IDEF5. Una vez explicado qué se entiende por ontología y su funcionalidad, se definen los componentes atómicos de IDEF5, y se introducen los conceptos básicos tanto del lenguaje esquemático como del lenguaje de elaboración. Señálese una vez más, antes de aproximarnos al estudio de IDEF5, que su puesta en práctica pone entre paréntesis la intensionalidad que Guarino propone como requisito. No obstante, al menos algunos de sus componentes y, por lo que más nos interesa, las relaciones, son declaradamente intensionales.

\section{Lenguaje IDEF5}

\subsection{Conceptos generales}

La técnica IDEF5 se basa en una gramática, dependiente en este caso tanto de anteriores investigaciones en el ámbito de la ontología y el conocimiento compartido, como de los conceptos más tradicionales de las teorías lógicas que entienden la verdad como adecuación a la realidad. De hecho, en el propio texto IDEF5 se menciona explícitamente su soporte en Knowledge Interchange Format, o los trabajos de Alfred Tarski y el segundo Wittgenstein.

La gramática IDEF5 contiene un conjunto de objetos, en sentido muy amplio, y unas instrucciones de combinación de éstos, a partir de los cuales se construye un lenguaje lógico que representa el compromiso ontológico para un espacio de dominio, o, si queremos hacer honor a la verdad, también para un dominio, en función del uso que el analista haga de la herramienta que tiene entre las manos. En lo que sigue describimos en filigrana estos objetos básicos de una ontología IDEF5:

1) Grupos: Traducimos "kinds" como "grupos", no como "clases" o "tipos", puesto que estos términos ya disponen de su espacio en IDEF5. El término "grupos", si bien más ambiguo, se adecúa razonablemente a lo que se quiere significar con "kind". En la terminología IDEF5 un grupo es, de manera informal, "un conjunto de individuos que comparten alguna serie de características distinguibles. De manera formal, los grupos son propiedades expresadas típicamente por nombres comunes". Si una ontología "filosófica" analiza el mundo en función de la naturaleza de las cosas que contiene, una ontología IDEF5 lo analiza en función de los roles que juegan esas cosas en un dominio dado. En consecuencia, una cosa, en términos generales, no ha de poseer una naturaleza para pertenecer a un grupo, sino más bien una serie de propiedades definitorias Por tales propiedades se entiende, en el lenguaje IDEF5, "características generales que son instanciables de manera múltiple, es decir, que pueden ser compartidas por diferentes

Scire. $8: 1$ (en.-jun. 2002) 55-86. 
objetos". Diremos, por tanto, que una cosa pertenece a un grupo si tiene cierto conjunto de tales propiedades. Por lo demás, debe quedar claro que estas propiedades no se atribuyen al grupo, sino a sus instancias.

Sin embargo, las propiedades definitorias de un grupo no son esenciales a sus instancias: una propiedad definitoria puede darse o no en una instancia, dependiendo del grupo en cuestión. De igual modo, el status de esencial de todas o algunas instancias de un grupo es independiente del status de sus propiedades definitorias. En consecuencia, las dimensiones "esencial" y "definitoria" de una clasificación IDEF5 son ortogonales. Además, los requisitos de pertenencia de una instancia a un grupo son más débiles que los de una ontología tradicional. El motivo es puramente práctico: no todas las instancias de un dominio son fácilmente reconocibles como tales, de modo que en ocasiones se debe confiar simplemente en la decisión de un experto en el dominio. Por tanto, sólo se requiere que, para una instancia dada de un grupo, dicha instancia tenga al menos una de las propiedades definitorias de tal grupo.Los grupos no son ni clases ni tipos, como ya se adelantó. No obstante, los tres conceptos son categoriales, es decir, indican algún modo de agrupamiento de individuos en categorías. Como consecuencia, los tres son instanciables de manera múltiple. Además, en el caso de los tipos y de los grupos, y a diferencia de las series y de las clases, aquéllos son intensionales, su identidad es independiente de su pertenencia: las instancias de un grupo pueden cambiar sin que el grupo mismo cambie. Pero, puesto que tanto la múltiple instanciabilidad como la intensionalidad son características distintivas de las propiedades, que ya ocupan un lugar en IDEF5, diremos, a efectos de simplificación, que los grupos son propiedades de un orden distinguible.

2) Propiedades y atributos: Un atributo se considera una función, es decir, un mapa que asigna a cada miembro de una serie de individuos un valor específico único. Por el contrario, una propiedad no mapea las cosas, sino que, más bien, es sólo una característica de una cosa: característica abstracta y general que algunos individuos tienen en común. En ocasiones, las cosas comparten atributos y propiedades, pero esto no es necesario en cualquier caso. Con frecuencia, las propiedades definitorias de un grupo serán independientes con respecto a un atributo. De aquí la utilidad, dentro de IDEF5, de distinguir entre ambos conceptos. Pero, desde un punto de vista práctico, mientras se está llevando a cabo el proceso de construcción de una ontología, resulta preferible utilizar el término neutro "característica", hasta que existan elementos de juicio suficientes para determinar si se está hablando de un atributo o de una propiedad.

3) Relaciones: Entre las demás características generales que los individuos pueden exhibir, aunque no individualmente, sino de manera conjunta, ocupan un lugar destacado las conexiones o asociaciones, o, en la terminología de IDEF5, las relaciones. Estas también son instanciables de manera múltiple e intensionales.

Scire. $8: 1$ (en.-jun. 2002) 55-86. 
Además, son típicamente binarias, aunque no existe límite al número de argumentos de una relación. En nuestro caso - como se dijo multidisciplinar, complejo, difuso...- una definición correcta y exhaustiva de relaciones resulta esencial.

4) Propiedades y relaciones de segundo orden: Los individuos son objetos de primer orden, y las propiedades y relaciones de objetos de primer orden son también de primer orden. Sin embargo, tanto las propiedades como las relaciones son ellas mismas objetos identificables; pero, puesto que se encuentran a un nivel de abstracción superior, pertenecen a un nivel lógico también superior y, por tanto, se clasifican como objetos de segundo orden. En tanto objetos, las propiedades y relaciones de primer orden pueden tener propiedades que se aplican a ellas, pero no a individuos; y, puesto que se aplican a objetos de segundo orden, estas propiedades también son de segundo orden. Además, los objetos de segundo orden pueden mantener relaciones entre ellos. Sin embargo, algunas relaciones de segundo orden pueden incluir individuos entre sus argumentos. La relación "instancia de", típica de IDEF5, es un ejemplo. A pesar de ser mixtas, estas relaciones son de igual modo de segundo orden. Una relación de segundo orden, por tanto, siempre incluye al menos una propiedad o relación de primer orden entre sus argumentos. Como se acaba de adelantar, las relaciones "instancia de" y "subgrupo de" se incluyen explícitamente en la gramática de IDEF5, con el fin de evitar las posibles ambigüedades derivadas de una relación "es un", que se excluye de la gramática.

5) Relaciones de subgrupo: La relación de subgrupo juega un papel relevante en la gramática de IDEF5, por el siguiente motivo: si K es un subgrupo de K', entonces cada instancia de K es una instancia de K'; aunque la inversa no es cierta: si una instancia de K es una instancia de otro K', de ello no se sigue que K sea un subgrupo de K'. La justificación de ello es que la relación de subgrupo es una relación necesaria: no es suficiente que, de hecho, cada K sea un K'; además, debe ser imposible que un K no sea un K'. Puesto que la noción de grupo incluye a las de tipo y clase, la noción de subgrupo también incluye a las de subtipo y subclase. Es decir, los tipos y clases son grupos con unas condiciones de pertenencia definidas, necesarias y suficientes. En consecuencia, un subtipo T de un tipo T' se adecúa a las condiciones de pertenencia de T'; es, entonces, un subgrupo de T'. Estas ocurrencias de la relación de subgrupo se conocen como subsunción de la descripción, ya que las condiciones de pertenencia de T' subsumen a las de T. Sin embargo, en aquellos casos en que un grupo no posee condiciones de pertenencia específicas, necesarias y suficientes, la relación de subgrupo sólo puede estipularse, pero no inferirse. Es decir, en los casos en los que sólo es posible declarar que un individuo dado es miembro de un cierto grupo K, la información asociada a K no proporciona ningún medio para determinar si otro grupo K' es además un subgrupo de $\mathrm{K}$. El motivo de ello es que, si no se determinan claramente las condiciones de pertenencia a un grupo dado $\mathrm{K}$, no existen garantías de que las condiciones

Scire. $8: 1$ (en.-jun. 2002) 55-86. 
de pertenencia de otro grupo K' sean suficientes para pertenecer a K. Por último, la noción de subgrupo también implica las de generalización y especialización, es decir, ocurrencias de la relación de subgrupo en las que se piensa de manera natural del subgrupo que es un caso especial de un concepto más general.

6) Partes, todos y grupos complejos. Los individuos no son simples, están a su vez compuestos de partes. El hecho de que se les considere desde uno u otro punto de vista depende de su relevancia para el dominio en cuestión. Es por ello por lo que IDEF5 incluye entre sus relaciones básicas primitivas la de "parte de", es decir, la que se mantiene entre un individuo y los objetos más complejos de los que forma parte. Cuando los miembros de un grupo se consideran como teniendo partes en una ontología dada, se dice de él que es un grupo complejo. La relación "parte de" se define como una ordenación parcial débil del dominio de individuos, y se caracteriza formalmente por completo por las propiedades de reflexividad y transitividad.

7) Procesos, estados y grupos de procesos. Las instancias de los grupos de un dominio están implicadas en procesos que suponen dos modos de cambio: cambio de grupo y cambio de estado. A diferencia de los estados, los grupos son agrupamientos contingentes dentro de un dominio. Es decir, lo que define a una característica de estado es que se trata de una característica accidental. Como los objetos individuales, los procesos también se agrupan en categorías generales. Así, pues, también los procesos son instanciables de manera múltiple e intensionales. En consecuencia, los procesos pueden considerarse grupos; pero, a diferencia de los grupos de individuos, los procesos son cosas que suceden: no sólo contienen otros objetos como partes, sino que ocurren en un intervalo de tiempo, y las cosas son "verdad" de los objetos del proceso al menos durante algunas partes de ese intervalo. IDEF5 permite la referencia explícita a los procesos, aunque de dos maneras diferentes, dependiendo del contexto. En primer lugar, si se hace referencia al carácter interno de un proceso $\mathrm{P}$, es decir, a la transición que implica a dos grupos, entonces se sigue el procedimiento IDEF3 (18) para la descripción de procesos, y P se describe en términos de los grupos que implica, sus propiedades y las relaciones relevantes que se mantienen entre las instancias de esos grupos cuando el proceso es instanciado. Por el contrario, si se hace referencia al modo en el que un grupo de proceso se relaciona lógicamente con otro grupo de proceso, con independencia de su estructura interna, entonces los grupos de proceso se caracterizan exactamente como grupos de objeto, según se ha explicado. En cualquier caso, cabe matizar que IDEF5 no se adentra en el análisis de procesos mediante el uso de lógicas temporales, la referencia a las cuales diríase natural en este caso. Tampoco se explica por qué no se profundiza en esta dirección, aunque nada en el método indique que las lógicas temporales resulten incoherentes con él. Sin embargo, en la medida en que no creemos que las alteraciones o las modificaciones ad hoc sean adecuadas en un método ya definido, no insistimos en esta mención a las tales lógicas no convencionales.

Scire. $8: 1$ (en.-jun. 2002) 55-86. 


\subsection{Proceso de desarrollo de una ontología IDEF5}

En la estructura misma del texto del informe IDEF5 se produce, en cierto sentido, una ruptura del carácter teórico del lenguaje, para introducir un punto de vista práctico o "de gestión”. Aunque esta ruptura puede parecer extraña, ya se adelantó que entre los objetos que intervienen en el proceso de desarrollo de una ontología se encuentran las personas, y que el olvido de esta premisa constituye la garantía de un fracaso. Por tanto, mencionamos en filigrana los pasos que habrían de darse para desarrollar una ontología.

\subsubsection{Organización y definición del proyecto}

Una ontología debe desarrollarse, idealmente, a partir de la formación de un equipo compuesto por un líder del proyecto, un ingeniero o analista del conocimiento, especializado en las técnicas IDEF5; un experto del dominio cuya ontología se va a desarrollar, en cuanto constituye la fuente primaria de información; y, por último, los revisores, que pueden ser simplemente lectores, o también comentaristas, es decir, estar autorizados para hacer críticas y modificaciones. Pueden existir más miembros en el equipo, pero las citadas son las figuras básicas.

En términos generales, el equipo debe establecer un contexto y propósito iniciales. La declaración del contexto delimita el área del dominio a la que se orienta el proyecto; es decir, el contexto declara el alcance del proyecto. Por su parte, el propósito proporciona una serie de criterios de exhaustividad para el proceso de descripción de la ontología. Así, pues, el propósito debe constar de declaraciones de objetivos, declaraciones de necesidades que la descripción debe satisfacer, y problemas que deben ser resueltos por la ontología resultante. Por supuesto, las declaraciones de propósito y contexto están sometidas a un proceso de revisión y retroalimentación durante el ciclo de vida del proyecto. La definición de propósito puede dividirse a su vez en dos partes: declaración de necesidades y declaración de objetivos. La primera debe identificar la fuente de la demanda y representar en proposiciones las motivaciones, declaradas explícitamente, del proyecto. La declaración de objetivos debe responder a cuestiones del estilo de: ¿qué decisiones debe apoyar la ontología? ¿Qué nivel de detalle se precisa en la ontología para tomar decisiones o resolver problemas? ¿A qué problemas debe responder el experto del dominio? ¿Quién utilizará la ontología una vez que esté disponible? Como se adelantó, el alcance define las fronteras del esfuerzo de desarrollo de la descripción, y especifica las partes del sistema que deben incluirse. Como parte del alcance, la especificación del nivel de detalle es normalmente un bloque de documentación con la forma de una serie de ejemplos.

A la hora de definir el proyecto, debe tenerse en cuenta que son individuos quienes lo llevan a cabo, y que individuos diferentes perciben el mundo de manera diferente, dependiendo de sus destrezas cognitivas y su conocimiento de base.

Scire. $8: 1$ (en.-jun. 2002) 55-86. 
Por tanto, la perspectiva, o el punto de vista en la capacidad para individuar, también debe reflejarse en el proyecto. También se sugirió más arriba que resulta imprescindible para el éxito de un proyecto incluir a las personas como objetos del dominio que se analiza. La descripción del proyecto adquiere finalmente forma física en un conjunto de formularios prediseñados.

\subsubsection{Colección de datos}

De manera intuitiva, puede decirse que las principales fuentes de datos son, no sólo los documentos relevantes, sino también los expertos del dominio. Sin embargo, uno de los problemas que plantean estos últimos es el hecho de que, si bien encuentran sencillo hacer explícito el conocimiento declarativo, tienen dificultades para hacer otro tanto con el conocimiento procedimental, en la medida en que la consciencia de éste decrece con la experiencia. En esta etapa, la colaboración entre los expertos del dominio y los analistas del conocimiento resulta fundamental. Los procedimientos habituales para recoger datos son la transcripción directa de los documentos fuentes, las entrevistas y análisis de protocolo con los expertos del dominio, y la observación introspectiva de determinados fenómenos o actividades en una organización. Puesto que los restantes son procedimientos suficientemente conocidos, nos detendremos brevemente en el análisis de protocolo.

Un protocolo es un modelo subyacente de discurso o proceso conductivo; por tanto, un protocolo significa, en términos prácticos, que un experto resuelve un problema mediante el uso de procedimientos y herramientas habituales. Un análisis de protocolo es el proceso de analizar un registro de discurso o un proceso conductivo. Existen dos tipos de análisis de protocolo: verbal, en el que el experto explica cómo resuelve el problema a medida que lo hace; y de movimiento, en el que el analista identifica éstos mediante el estudio de la eficacia del proceso. Todo el proceso de colección de datos, por supuesto, se encuentra también documentado de manera gráfica en los correspondientes formularios.

\subsubsection{Análisis de datos}

El objetivo de esta tarea es analizar el material fuente para construir una caracterización inicial de la ontología, e implica, por regla general, tres actividades: 1) listado de objetos, en sentido amplio, de potencial interés para el dominio; 2) identificación de los objetos que constituyen la frontera de la ontología, aunque esta frontera tenga que ser dibujada de nuevo posteriormente; y 3) búsqueda e individuación de sistemas internos dentro de las fronteras de la descripción, actividad que resulta de particular relevancia, en la medida en que los sistemas son colecciones de objetos físicos y/o conceptuales que operan en conjunto para lograr un objetivo común. La organización de ontologías mediante sistemas del dominio proporciona una estructura conceptual clave para un subsiguiente análisis del conocimiento.

Scire. $8: 1$ (en.-jun. 2002) 55-86. 


\subsubsection{Desarrollo de una ontología inicial}

En esta etapa se lleva a cabo el proceso de desarrollo de proto-conceptos, es decir, proto-grupos, proto-propiedades, proto-atributos y proto-relaciones. El prefijo "proto" sugiere que estos conceptos son tentativos y se encuentran sujetos a revisión y prueba antes de pasar a ser definitivos. IDEF5 explica detalladamente, y como hasta ahora de manera gráfica, el procedimiento para la elaboración de proto-conceptos. De igual modo, comienza a utilizarse rudimentariamente el llamado lenguaje esquemático, que examinaremos en un instante.

\subsubsection{Refinamiento y validación de la ontología}

El objetivo de esta etapa es refinar los proto-conceptos, para confirmar su autenticidad y convertirlos en definitivamente válidos. El proceso de refinamiento es básicamente un proceso de validación deductiva. Las estructuras de la ontología se "instancian" o testean con datos reales, y el resultado de la instanciación se contrasta con la estructura de la ontología. Si durante la comparación se produce un desajuste, éste debe resolverse de la manera adecuada. De igual modo, se incorporan los anteriores refinamientos de la ontología inicial, para obtener una ontología "validada".

\subsection{Los lenguajes IDEF5}

Si entendemos ontología como la caracterización explícita —es decir, lingüística— detallada de los componentes, en sentido amplio, de un determinado dominio, entonces, como resulta obvio, debiéramos disponer de un lenguaje que expresara esta caracterización detallada. IDEF5 identifica dos motivos básicos por los que se debe disponer de un lenguaje para la transcripción de una ontología: porque un lenguaje constituye el medio para capturar y almacenar el conocimiento, y porque proporciona el formato adecuado para presentar ese conocimiento previamente adquirido. Además, IDEF5 insiste en la necesidad de que la estructura representativa sea rica y expresiva, en primer lugar porque el conocimiento adquirido constituye la base para adquirir nuevo conocimiento, y, en segundo, porque no existe ninguna garantía previa de las estructuras de representación que se necesitarán para describir la ontología de un dominio dado. No obstante, es evidente que la riqueza del lenguaje no debe hacer difícil su uso; es decir, debe mantener tanto el paralelismo con las actividades cognitivas implicadas en el proceso de desarrollo de la ontología, como la sinergia con el procedimiento de desarrollo de ésta.

IDEF5 propone dos lenguajes, no alternativos, sino sucesivos y/o complementarios, en función de la fase de desarrollo de la ontología en la que se encuentre el analista, y por supuesto coherentes entre sí. Son el lenguaje esquemático, marcadamente visual y destinado a facilitar la comunicación; y el lenguaje de elaboración, textual y basado en la lógica de primer orden y la filosofía KIF. El lenguaje esquemático dispone de un léxico muy pequeño, a partir del cual se construyen los

Scire. $8: 1$ (en.-jun. 2002) 55-86. 
esquemas que representan visualmente la ontología. Entendemos por "léxico", en el contexto IDEF5, la serie de símbolos básicos de un lenguaje. Por esquema entendemos un diagrama de conexiones, construído a partir del léxico del lenguaje esquemático IDEF5, de acuerdo con las recomendaciones sintácticas de éste. Puesto que los esquemas son interpretaciones de información, IDEF5 debe dotarlos de reglas semánticas que proporcionen sentido unívoco a tales esquemas. Estas reglas semánticas interpretan los esquemas básicos del lenguaje y, a partir de aquí, se aplican recursivamente a esquemas cada vez más complejos. Cada esquema básico tiene sólo una semántica por defecto, que puede ser obviada al pasar al lenguaje de elaboración. El motivo de ello es que, como se apuntó, el lenguaje esquemático no ha sido ideado para diseñar la ontología, sino para servir de asistente a este diseño. Su función primordial consiste en registrar, de manera más bien rústica, los grupos de cosas en el dominio, sus propiedades, y las relaciones entre los objetos de los grupos y los grupos mismos. Sin embargo, su semántica por defecto no tiene por qué expresar necesariamente la información deseada, de tal manera que los analistas pueden pasarla por alto en el lenguaje de elaboración. Por otra parte, puesto que la realidad es múltiple y compleja, la semántica por defecto de los esquemas básicos muestra los significados que la experiencia ha mostrado como más útiles, aunque éstos son susceptibles de revisión en el lenguaje de elaboración.

A tenor de lo dicho anteriormente, cabe preguntarse por qué no proponer un solo lenguaje completo de primer orden. IDEF5 responde a esta reserva de manera triple: 1) un lenguaje gráfico sencillo resulta de utilidad para los no especialistas; 2) pero, en opinión de los expertos, no contribuye a mejorar ni el aprendizaje ni el uso; y 3) además, no es procesable por un ordenador, que precisa del lenguaje lógico textual que viene a corresponderse con el lenguaje de elaboración.

\subsubsection{El lenguaje esquemático IDEF5}

En filigrana, los esquemas IDEF5 se construyen mediante la unión de símbolos gráficos básicos IDEF5 de diferentes maneras. Los esquemas básicos IDEF5 son los diagramas más pequeños que pueden construirse mediante este procedimiento. La sintaxis de los esquemas básicos es tan simple como la conexión de círculos mediante símbolos de relación y proto-relación.

Con respecto a la semántica, y al menos en lo que se refiere a los esquemas de primer orden, es decir, aquellos que reflejan relaciones entre objetos reales, debe tomarse la precaución de interpretarlos en sentido débil, precisamente por cuanto se refieren a objetos reales. Es decir, los esquemas básicos de primer orden debieran indicar sólo lo que es posible o permisible en un cierto dominio. No obstante, el concepto de posibilidad en los esquemas básicos IDEF5 es más fuerte que el de mera posibilidad lógica, puesto que debe reflejar la naturaleza real de un dominio dado, el modo en que las cosas son el dominio, siendo iguales a otras

Scire. $8: 1$ (en.-jun. 2002) 55-86. 
cosas; no el modo en que podrían ser las cosas bajo cualquier circunstancia imaginable. Puesto que en nuestro caso hemos optado por una acepción intensional de ontología, esta condición, que contradice las que establecimos en un principio, es ejemplo del margen de indefinición en que se encuentra IDEF5. En esta situación, sí creemos que debemos ser estrictos y aceptar la restricción mencionada únicamente si se está trabajando con el lenguaje esquemático y en un área única y bien definida. Bajo ninguna circunstancia aceptaremos la restricción si se está utilizando el lenguaje de elaboración y en contextos multidisciplinares.

La semántica de los esquemas de segundo orden resulta mucha más perfilada, puesto que representa grupos directamente, no las instancias de éstos. En este caso, la semántica por defecto no está calificada. El motivo de ello es que el esquema de segundo orden no representa el modo en que dos cosas pueden ser en el dominio, sino el modo en que dos grupos están relacionados de hecho. Ello se debe a la circunstancia de que las aserciones de segundo orden se refieren a la naturaleza de los grupos en cuestión, y no son dependientes, en general, de las contingencias de un dominio. El lenguaje esquemático IDEF5 especifica una biblioteca de ocho tipos de esquemas que, por motivos de espacio, simplemente se enumeran: a) esquemas básicos de primer orden, subdivididos en esquemas de relación binaria, existenciales y n-arios de primer orden; b) esquemas complejos de primer orden, o, en general, convenciones que no implican símbolos de proceso; c) esquemas de segundo orden; d) esquemas de relación; e) referentes, o mecanismos para enlazar un concepto IDEF5 con otro concepto de cualquiera de las otras técnicas IDEF; f) esquemas de composición o, básicamente, aquellos que se basan en la relación "parte de"; g) esquemas de clasificación, que se corresponden, en filigrana, con aquellos que utilizan relaciones del tipo "es un", "tipo de", "grupo de"; y e) esquemas de estado de objeto, divididos en esquemas básicos de transición, de transición instantánea, de clasificación y de composición.

\subsubsection{El lenguaje de elaboración IDEF5}

El lenguaje de elaboración, como se dijo, no es una alternativa al lenguaje esquemático. Más bien, éste último complementa o sirve de borrador o ayuda gráfica a aquél, que es en realidad el lenguaje textual en el que debe elaborarse una ontología. El lenguaje de elaboración es, por tanto, un lenguaje estructurado, expresivamente rico y fundado teóricamente. La usanza de su sintaxis y de su alfabeto está basada en el Knowledge Interchange Format, es decir, la sintaxis utiliza una notación de prefijos y paréntesis para delimitar expresiones, y el alfabeto consta de caracteres alfanuméricos y signos de puntuación normalizados. Una "cadena" es una secuencia finita de caracteres y espacios en blanco encerrados entre comillas. Esta dependencia de KIF, al tiempo que se pretende elaborar un método de desarrollo de ontologías basado en componentes intensionales es,

Scire. $8: 1$ (en.-jun. 2002) 55-86. 
a nuestro juicio, la causa primera de aquel margen de indefinición y de contradicción ya mencionado hasta la saciedad.

De igual modo en filigrana, el lenguaje de elaboración consta de seis recursos, todos ellos definidos formal y lógicamente, excepto la noción de "palabra", que es un primitivo del lenguaje: 1) constantes, o palabras que no son ni operadores ni variables, es decir, que denotan objetos del universo y de las que existen quince definidas en el lenguaje; 2) variables, o instancias únicas de una constante, que pueden ser variables individuales — precedidas por el carácter ? - o de predicado —precedidas por el carácter \#—; 3) operadores, o palabras y caracteres reservados que se utilizan para formar expresiones complejas, de los cuales existen tres bloques - cinco de definición, seis de término, y los de sentencia, que incluyen tanto los operadores lógicos habituales, como dos operadores modales y una biblioteca de operadores específicos de IDEF5-; 4) términos, que se utilizan para denotar objetos, y están divididos en ocho categorías; 5) definiciones, que son expresiones utilizadas para definir constantes de individuo, de relación o de función, y que pueden ser completas o incompletas, dependiendo de que la información que proporciona acerca de la constante sea completa o limitada; y 6) sentencias, o expresiones de algún hecho acerca de las constantes de la ontología. Existen siete tipos de sentencias: constantes lógicas, ecuaciones, desigualdades, sentencias de relación, sentencias lógicas, sentencias cuantificadas y sentencias específicas de IDEF5. Con las constantes, las variables y los operadores se construyen las sentencias IDEF5.

\subsubsection{Sentencias especificas de IDEF5}

Las sentencias específicas de IDEF5 permiten la introducción de constantes de conformidad con la estructura conceptual del método IDEF5. Existen once categorías de este tipo de sentencias, correspondiendo cada una de ellas a un concepto IDEF5: 1) constructos de ontología, que expresan información sobre una ontología, y de los que existen ocho; 2) constructos de grupo (nueve tipos), que expresan información sobre un grupo: 3) constructos de propiedad (tres tipos), que se utilizan para dar información sobre una propiedad; 4) constructos de individuo (tres tipos), que dan información sobre un individuo; 5) constructos de atributo (tres tipos), que se utilizan para expresar información sobre atributos; 6) constructos de relación (cuatro tipos), de especial interés para nosotros, que se utilizan para proporcionar información sobre relaciones, y que pueden ser de relación para declarar relaciones, de exponente de relación para declarar su exponente o aridez, de tipo de argumento para declarar el grupo de individuos que pueden estar en ella, y de descripción de la relación para describir una relación; 7) constructos de función (cuatro tipos), que se utilizan para proporcionar información sobre funciones; 8) constructos de fuente (nueve tipos), que se utilizan para dar información sobre los materiales fuente; 9) constructos de mención de fuente

Scire. $8: 1$ (en.-jun. 2002) 55-86. 
(cuatro tipos), que se usan para proporcionar información sobre las menciones de fuente; 10) constructos de término de ontología (tres tipos), que se utilizan para ofrecer información sobre los términos de la ontología; 11) cconstructos de nota (tres tipos), que se usan para dar información sobre las notas.

Por último, IDEF5 predefine diez relaciones que pueden ser utilizadas de principio para la construcción de sentencias: parte-de, transiciones-a, transiciones-durante, transiciones-instantáneas-a, transiciones-instantáneas-durante, transiciones-fuertes-a, transiciones-instantáneas-fuertes-a, transiciones-instantáneas-fuertes-durante, en-estado-durante.

\subsubsection{Biblioteca de relaciones IDEF5}

El de relación es un concepto especialmente relevante en cualquier ontología, $\mathrm{y}$, en nuestro caso, hemos insistido en la utilidad que nos reporta. En IDEF5 juega un importante papel, tanto en el lenguaje esquemático como en el de elaboración. En el entorno en el que nos desenvolvemos - la organización de la información en una institución compleja-, una definición bien formada y hasta donde sea posible exhaustiva de los distintos tipos de relación resulta imprescindible. IDEF5 incorpora una biblioteca de relaciones, en un estilo similar a lo que sería, por analogía, una biblioteca de rutinas en programación; es decir un conjunto de relaciones de uso frecuente, que ya han sido formalmente definidas, y que el analista puede utilizar directamente. Las definiciones de estas relaciones están escritas en el lenguaje de elaboración y han sido axiomatizadas, entendiendo axioma de acuerdo al uso que de este término se hace en IDEF5: una caracterización precisa de la lógica de un término o de una serie de términos relacionados, que han sido declarados en el lenguaje de elaboración IDEF5, y que expresa típicamente una restricción sobre los objetos denotados por el término axiomatizado. La biblioteca de relaciones, además, es extensible, en la medida en que se puede incorporar a ella cualquier nueva relación que haya sido definida formalmente. Las relaciones se agrupan en siete categorías: relaciones de clasificación, relaciones meronímicas, relaciones temporales, relaciones espaciales, relaciones de influencia, relaciones de dependencia y relaciones de caso. Todas ellas, como se dijo, han sido, no sólo informalmente explicadas, sino también formalmente definidas y axiomatizadas, lo cual incluye la caracterización de cada una de ellas. Esta biblioteca, si bien no se incorpora al método IDEF5 en sentido estricto, puesto que se encuentra abierta y tiene el status de apéndice, constituye una herramienta de utilidad incuestionable en el proceso de elaboración de una ontología.

\section{Herramientas para el desarrollo de una ontología IDEF5}

Como se dijo, para el uso del método IDEF5 sólo se requiere papel y lápiz. Sin embargo, resulta evidente la utilidad de trasladarlo a una herramienta informatiza-

Scire. $8: 1$ (en.-jun. 2002) 55-86. 
da. En el curso del tiempo, se han desarrollado numerosas aplicaciones informáticas, se han creado grupos de usuarios con sus correspondientes foros de discusión, etc. Algunas de estas aplicaciones son de libre utilización y otras comerciales. Pero los productos desarrollados por la empresa KBSI (19), desgraciadamente pertenecientes al segundo grupo, parecen haber obtenido el beneplácito y un cierto grado de oficialización por parte de los creadores de los métodos IDEF. Existen, por supuesto, soluciones más avanzadas, especialmente aquellas que combinan la organización del conocimiento con las especificaciones XML (20), pero, insistimos, no son las soluciones que precisamos en nuestro contexto. KBSI ha desarrollado una serie de herramientas —-ProSim, ProCap, SmartER, AIOWIN...—, ninguna de las cuales se corresponde unívocamente con un solo método IDEF, pero que pueden utilizarse en conjunción para llevar a cabo distintos tipos de proyectos, incluídos, claro está, los de elaboración de ontologías. El producto —o la combinación de ellos - que se utilice depende de las necesidades del analista y del material que tiene entre las manos. No es nuestra intención vender un producto. Ya hemos dicho que existen otros. No obstante, la familia KBSI es de utilización simple y válida en múltiples contextos, y el esfuerzo que se ha realizado para adecuarse a los métodos IDEF ha resultado en un alto nivel de coherencia entre el método y la herramienta. Por ello la familia KBSI nos parece realmente útil, a pesar del grave inconveniente de que hay que pagar por ella, cuando otras aplicaciones son de uso gratuíto. En cualquier caso, ésta es sólo una sugerencia, y la decisión última depende de las necesidades y los recursos del analista.

\section{Notas}

(1) http://www.ontoknowledge.org/oil/

(2) http://www.cs.umd.edu/projects/plus/SHOE/

(3) http://smi-web.stanford.edu/projects/protege/

(4) Gruber, Tom: What is an ontology? (http://www-ksl.stanford.edu/kst/what-is-anontology.html)

(5) http://www.idef.com/Downloads/pdf/idef0.pdf

(6) Gruber: Ibidem

(7) A Glossary of Ontology Terminology (http://www-kslsvc.stanford.edu:5915/doc/frame-editor/glossary-of-terms.html)

(8) IDEF5 Method Report (http://www.idef.com/Downloads/pdf/Idef5.pdf)

(9) Resource Description Framework : (RDF) Schema Specification 1.0 (http://www.w3.org/TR/rdf-schema/)

(10) http://www.cyc.com/

(11) http://business.semanticweb.org/swws-bof-discussion.html

(12) http://www.c3i.osd.mil/bpr/bpred/mhome.htm

(13) http://www.c3i.osd.mil/bpr/bprcd/0024.htm

Scire. $8: 1$ (en.-jun. 2002) 55-86. 
(14) http://www.c3i.osd.mil/bpr/bprcd/mhome.htm

(15) http://www.idef.com/Downloads/pdf/bpr.pdf

(16) http://212.73.32.211/hosting/0003e/jmfhevia/guia.htm

(17) http://www.idef.com/Downloads/free_downloads.html

(18) http://www.idef.com/Downloads/pdf/Idef3_fn.pdf

(19) http://www.kbsi.com

(20) Véase, a modo de ejemplo y de rastreo, http://www.metaintegration.net

\section{Referencias}

Aerts, D.; Apostel, L.; De Moor, B.; Hellemans, S.; Maex, E.; Van Belle, H.; Van Der Veken, J. (1994). Worldviews: From Fragmentation to Integration. Brussels: VUB Press, 1994. URL: <http://pespmc1.vub.ac.be/CLEA/reports/worldviewsbook .html>. Consultado: 2002-12-08.

Allen, J.; Lehrer, N. (1992). DRAFT of the DARPA/Rome Laboratory Planning and Scheduling Initiative Knowledge Representation Specification Language (KRSL): Reference Manual. Version 2.0.1. [s.1.]: ISX Corporation, 1992. URL: <http://wwwksl.stanford.edu/knowledge-sharing/papers/\#krsl>. Consultado: 2002-12-08.

Ambite, J.-L.; Arens, Y.; Hovy, E.H.; Philpot, A.; Gravano, L.; Hatzivassiloglou, V.; Klavans, J.L. (2001). Simplifying Data Access: The Energy Data Collection Project. // IEEE Computer. 34: 2 (February 2001). 47-54. URL: <http://www.isi.edu/ ambite/ EDCIEEE-Computer-2001.pdf>. Consultado: 2002-12-08.

Andersen, W.A.; Hendler, J.A.; Evett, M.P.; Kettler, B.P. (1994). Massively Parallel Matching of Knowledge Structures. // Kitano, H.; Hendler J. (eds.). Massively Parallel Artificial Intelligence. Menlo Park: AAAI/The MIT Press, 1994. URL: < http:// citeseer.nj.nec.com/cache/papers/cs/660/http:zSzzSzwww.cs.umd.eduzSzprojectszSzpl uszSzParkazSzparka-mpai.pdf/andersen94massively.pdf > . Consultado: 2002-12-08.

Arens, Y.; Hovy, E.H. (1995). The Design of a Model-Based Multimedia Interaction Manager. // AI Review 8: 3 (1995). URL: <http://www.isi.edu/natural-language/people/ hovy/papers/95arenshovy.pdf $>$. Consultado: 2002-12-08.

Arens, Y.; Hovy, E.H.; Van Mulken, S. (1993). Structure and Rules in Automated Multimedia Presentation Planning. // Proceedings of the International Joint Conference on Artificial Intelligence: IJCAI-93. France: IJCAI, 1993. URL: < http://citeseer.nj. nec.com/cache/papers/cs/7242/http:zSzzSzwww.isi.eduzSznatural-languagez SzmultimediazSzmm-planning.pdf/arens93structure.pdf >. Consultado: 2002-12-08.

Arens, Y.; Hovy, E.H. Vossers, M. (1993). Describing the Presentational Knowledge Underlying Multimedia Instruction Manuals. // Maybury, M. (ed). Intelligent Multimedia Interfaces. Cambridge: MIT Press, 1993. URL: <http://www.isi.edu/ naturallanguage/projects/multimedia-com.html>. Consultado: 2002-12-08.

Artale, A.; Franconi, E.; Guarino, N. (1996). Open Problems for Part-Whole Relations. // Proceedings of 1996 International Workshop on Description Logics :DL-96. Cambridge, AAAI Press, 1996. 70-73. URL: <http://www.acsu.buffalo.edu/ ryankohl/1996ArtaleFranconiGuarino.doc>. Consultado: 2002-12-08.

Artale, A.; Franconi, E.; Guarino, N.; Pazzi, L. (1996). Part-Whole Relations in Object-Centered

Scire. $8: 1$ (en.-jun. 2002) 55-86. 
Systems: an Overview. // Data and Knowledge Engineering. 20: 3 (1996) 347-383. URL: <http://citeseer.nj.nec.com/cache/papers/cs/15850/http:zSzzSzwww.dblab. ntua.grzSzzCz7EdwqzSzdke-96_dwq.pdf/artale96partwhole.pdf >. Consultado: 2002-12-08.

Borgid, A. (1996). On the Relative Expressiveness of Description Logics and Predicate Logics. [s.1.]: Deptartment of Computer Science, Rutgers University, 1996. URL: < http://citeseer.nj.nec.com/cache/papers/cs/557/http:zSzzSzmnemosyne.itc.it:1024zSzdlz SzcoursezSzarticleszSzdl-vs-fol.pdf/borgida96relative.pdf > . Consultado: 2002-12-08.

Borgo, S.; Guarino, N.; Masolo C. A. (1996a). Pointless Theory of Space based on Strong Connection and Congruence. // Carlucci Ailello, L.; Doyle, J.; Shapiro S. C. (eds.). Principles of Knowledge Representation and Reasoning (KR96): Proceedings of the Fifth International Conference (KR '96). Boston: Morgan Kaufmann, 1996. 220-229. URL: < http:// citeseer.nj.nec.com/cache/papers/cs/297/http:zSzzSzwww.ladseb.pd.cnr.itzSzinforzSzOntol ogyzSzPaperszSzKR96.pdf/a-pointless-theory-of.pdf > . Consultado: 2002-12-08.

Borgo, S.; Guarino, N.; Masolo, C. (1996b). Stratified Ontologies: the Case of Physical Objects. // Proceedings of ECAI-96 Workshop on Ontological Engineering. [s.1.]: ECAI, 1996. URL: 〈http://www.ladseb.pd.cnr.it/infor/Ontology/ Papers/StratOntologies.pdf>. Consultado: 2002-12-08.

Borgo, S.; Guarino, N.; Masolo, C. (1997a). Qualitative Spatial Modelling Based on Parthood, Strong Connection and Congruence: LADSEB-CNR Internal Report 03/97. Padova: LADSEB, 1997. URL: 〈http://www.ladseb.pd.cnr.it/infor/Ontology/ Papers/QSM.pdf〉. Consultado: 2002-12-08.

Borgo, S.; Guarino, N.; Masolo, C. (1997b). An Ontological Theory of Physical Objects. // Proceedings of Qualitative Reasoning 11th International Workshop. Pavia: IAN-CNR, 1997. 223-231. URL: <http://ontology.ip.rm.cnr.it/Papers/QR97.pdf>. Consultado: 2002-12-08.

Borgo, S.; Guarino, N.; Masolo, C.; Vetere, G. (1997). Using a Large Linguistic Ontology for Internet-Based Retrieval of Object-Oriented Components. // Proceedings of 1997 Conference on Software Engineering and Knowledge Engineering. Madrid: Knowledge Systems Institute, Snokie, 1997. 528-534. URL: <http:// www.ladseb.pd.cnr.it/infor/ Ontology/Papers/SEKE97.doc>. Consultado: 2002-12-08.

Borgo, S.; Masolo, C. (2001). Mereogeometries: a Semantic Comparison: LADSEB-CNR Techical Report 02/01. Padova: LADSEB, 2001. URL: <http://www.ladseb.pd.cnr.it/ infor/Ontology/Papers/TR-02-2001.pdf >. Consultado: 2002-12-08.

Broekstra, J.; Klein, M. Adding formal semantics to the Web: building on top of RDF Schema Business Object Ontologies. URL: <http://www.ladseb.pd.cnr.it/infor/ ontology/BusinessObjectsOntology.html>. Consultado: 2002-12-08.

Carrara, M.; Guarino, N. (1999). Formal Ontology and Conceptual Analysis: A Structured Bibliography. V2.5. [s.1.: s.n.], 1999. URL: <http://www.ladseb.pd.cnr.it/infor/ ontology/Papers/Ontobiblio/TOC.html>. Consultado: 2002-12-08.

Cutkosky, M.; Engelmore, R.; Fikes, R.; Gruber, T.; Genesereth, M.; Mark, W.; Tenenbaum, J.; Weber, J. (1993). PACT: An experiment in integrating concurrent engineering systems. // IEEE Computer (January 1993). URL: < http://citeseer.nj.nec.com/cache/ papers/cs/148/ http:zSzzSzksl.stanford.eduzSzknowledge-sharingzSzpaperszSzpact. pdf/cukosky93pact.pdf > . Consultado: 2002-12-08.

Decker, S. Van Harmelen, F.; Broekstra, J.; Erdmann, M.; Fensel, D.; Horrocks, I.; Klein, M.; 
Melnik, S. The Semantic Web: on the respective Roles of XML and RDF. URL: <http://www.ontoknowledge.org/oil/downl/IEEE00.pdf>. Consultado: 2002-12-08.

Dorr, B.; Hendler, J.; Blanksteen, S.; Migdalof, B. (1994). Use of Lexical Conceptual Structure for Intelligent Tutoring. Maryland; Department of Computer Science, University of Maryland at College Park, 1994. URL: <http://www.umiacs.umd.edu/labs/CLIP/ Abstracts/dorr_hendler_blanksteen_migdalof_94.html>. Consultado: 2002-12-08.

KRSS working group of the DARPA Knowledge Sharing Effort. Draft of the specification for Description Logics produced by the KRSS working group of the DARPA Knowledge Sharing Effort. [s.l.: s.n.], 1993. URL: <http://www-ksl.stanford.edu/knowledgesharing/papers/>. Consultado: 2002-12-08.

Erdmann, M.; Studder, R. (1999). Ontologies as Conceptual Models for XML Documents. Karlsruhe: Institut für Angewandte Informatik und Formale Beschreibungsverfahren, University of Karlsruhe. URL: <http://sern.ucalgary.ca/KSI/KAW/KAW99/ papers/Erdmann1/erdmann.pdf >. Consultado: 2002-12-08.

Erol, K.; Nau, D.; Hendler, J. (1994a). UMCP: A Sound and Complete Planning Procedure for Hierarchical Task-Network Planning. // AIPS-94. [s.1.: s.n.], 1994. URL: <http:// citeseer.nj.nec.com/cache/papers/cs/660/http:zSzzSzwww.cs.umd.eduzSzuserszSzkutlu hanzSzPaperszSzAIPS-94.pdf/umcp-a-sound-and.pdf>. Consultado: 2002-12-08.

Erol, K.; Nau, D.; Hendler, J. (1994b). HTN Planning: Complexity and Expressivity. // AAAI94, Menlo Park: The AAAI Press, 1994. URL: <http://citeseer.nj.nec.com/ cache/papers/cs/660/http:zSzzSzwww.cs.umd.eduzSzuserszSzkutluhanzSzPaperszSzA AAI-94.pdf/htn-planning-complexity-and.pdf >. Consultado: 2002-12-08.

Erol, K.; Nau, D.; Hendler, J.; Tsuneto, R. (1995). A Critical Look at Critics in HTN Planning. // IJCAI-95. [s.1.: s.n.], 1995. URL: <http://www.cs.umd.edu/ nau/papers/criticsijcai95.ps>. Consultado: 2002-12-08.

Evett, M.P.; Andersen, W.A.; Hendler, J.A. (1993). Massively Parallel Support for Computationally Effective Recognition Queries. // Proceedings of the Eleventh National Conference on Artificial Intelligence (AAAI-93). Menlo Park: AAAI Press, 1993.

Evett, M.P.; Andersen, W.A.; Hendler, J.A. (1993). Massively Parallel Support for Efficient Knowledge Representation. // Proceedings of the Thirteenth International Joint Conference on Artificial Intelligence (IJCAI-93). Denver: Morgan Kaufmann, 1993.

Evett, M.P.; Andersen, W.A.; Hendler, J.A. (1994). Providing Computational Effective Knowledge Representation via Massive Parallelism. // Kanal, L.; Kumar, V.; Kitano, H.; Suttner, C. (eds.). Parallel Processing for Artificial Intelligence. [s.l.]: Elsevier Science Publishers, 1994. URL: <http://www.cs.umd.edu/projects/plus/Parka/parka-pubs.html>. Consultado: 2002-12-08.

Evett, M.P.; Hendler, J.A.; Spector, L. (1994). Parallel Knowledge Representation on the Connection Machine. // Journal of Parallel and Distributed Computing. 22 (1994) 168-184.

Fensel, D. A. (2000a). Unifying Framework for the Knowledge Web. // Proceedings of the Workshop on Applications of Ontologies and Problem-solving Methods: 14th European Conference on Artificial Intelligence: ECAI-00: Berlin, Germany, August 20-25, 2000. [s.1.: s.n.], 2000. URL: 〈http://www.cs.vu.nl/ dieter/ftp/spool/upml.oil.pdf>. Consultado: 2002-12-08.

Fensel, D. (2000b). OIL in a nutshell. // Dieng, R. et al. (eds.). Knowledge Acquisition, Modeling, and Management: Proceedings of the European Knowledge Acquisition

Scire. $8: 1$ (en.-jun. 2002) 55-86. 
Conference (EKAW-2000): Lecture Notes in Artificial Intelligence. [s.1.]: LNAI, Springer-Verlag, 2000. URL: <http://www.cs.vu.nl/ ontoknow/oil/downl/ oilnutshell.pdf>. Consultado: 2002-12-08.

Fensel, D.; Van Harmelen, F.; Horrocks, I. (2002). OIL: A Standard Proposal for the Semantic Web: Deliverable 0 in the European IST project OnToKnowledge. URL: <http://www.ontoknowledge.org/oil/downl/otk.del02.pdf>. Consultado: 2002-12-08.

Fikes, R.; Cutkosky, M.; Gruber, T.; Van Baalen, J. (1991). Knowledge Sharing Technology Project Overview: Technical Report KSL 91-71. Stanford: University, Knowledge Systems Laboratory, 1991.

Filatova, E.; Hovy, E.H. (2001). Assigning Time-Stamps to Event-Clauses. // Proceedings of the Workshop on Temporal and Spatial Reasoning at the ACL-01 Conference. Toulouse: [s.n.], 2001. URL: <http://www.isi.edu/natural-language/projects/webclopedia/ pubs/01timestamping.pdf>. Consultado: 2002-12-08.

Finin, T.; McKay, D.; Fritzson, R. (eds.). (1992). An overview of KQML: A Knowledge Query and Manipulation Language. [s.1.]: The KQML Advisory Group, 1992. URL: <http://www-ksl.stanford.edu/knowledge-sharing/papers/\#kqml-overview>. Consultado: 2002-12-08.

Finin, T.; Weber, J.; Wiederhold, G.; Genesereth, M.; Fritzson, R.; McKay, D.; McGuire, J.; Pelavin, P.; Shapiro, S.; Beck, C. (1993). Specification of the KQML AgentCommunication Language: Technical Report EIT TR 92-04. Palo Alto: Enterprise Integration Technologies, 1993. URL: <http://www-ksl.stanford.edu/knowledgesharing/papers/\#kqml-spec>. Consultado: 2002-12-08.

Gangemi, A.; Guarino, N.; Masolo, C.; Oltramari, A. (2001a). Understanding top-level ontological distinctions. // Proceedings of IJCAI 2001 workshop on Ontologies and Information Sharing. Padova: LADSEB, 2001. URL: <http://www.ladseb.pd.cnr.it/ infor/Ontology/Papers/IJCAI2001ws.pdf>. Consultado: 2002-12-08.

Gangemi, A.; Guarino, N.; Masolo, C.; Oltramari, A. (2001b). Understanding Top-level Ontological Distinctions: LADSEB-CNR Int. Rep. 04/2001. Padova: LADSEB, 2001. URL: <http://www.cs.umbc.edu/771/papers/IJCAI2001final.pdf>. Consultado: 2002-12-08.

Gangemi, A.; Guarino, N.; Oltramari, A. (2001). Conceptual Analysis of Lexical Taxonomies: The Case of WordNet Top-Level. // Proceedings of FOIS2001. Padova: LADSEB, 2001. URL: <http://www.ladseb.pd.cnr.it/infor/Ontology/Papers/FOIS2001-Final.pdf>. Consultado: 2002-12-08.

Genesereth, M. R.; Fikes, R. E. (eds.). (1992). Knowledge Interchange Format, Version 3.0: Reference Manual: Technical Report Logic-92-1. Standford: Computer Science Department, Stanford University, 1992. URL: <http://logic.stanford.edu/kif/Hypertext/ kif-manual.html>. Consultado: 2002-12-08.

Germann, U. (1998a). Making Semantic Interpretation Parser-Independent. // Proceedings of the 4th AMTA Conference. [s.1.: s.n.], 1998. URL: <http://www.isi.edu/natural-language/ people/germann/publications/u.germann.amta.1998.pdf>. Consultado: 2002-12-08.

Germann, U. (1998b). Visualization of Protocols of the Parsing and Semantic Interpretation Steps in a Machine Translation System. // COLING-ACL Workshop on Content Visualization and Intermedia Representations. [s.1.: s.n.], 1998. URL: <http://www.isi.edu/ natural-language/people/germann/ publications/ContentVisualization.pdf > . Consultado: 
2002-12-08.

Germann, U. (2001). Building a Statistical Machine Translation System from Scratch: How Much Bang Can We Expect for the Buck. // Proceedings of the Data-Driven MT Workshop of ACL-01. [s.1.: s.n.], 2001. URL: <http://www.isi.edu/natural-language/people/germann/ publications/germann-acl-2001-DDMT-workshop.pdf>. Consultado: 2002-12-08.

Germann, U.; Jahr, M.; Knight, K.; Marcu, D.; Yamada, K. (2001). Fast Decoding and Optimal Decoding for Machine Translation. // Proceedings of ACL-01. [s.1.: s.n.], 2001. URL: <http://www.isi.edu/natural-language/projects/rewrite/decoder.pdf >. Consultado: 2002-12-08.

Gruber, T. R. (1990). The Role of Standard Knowledge Representation for Sharing Knowledge-Based Technology: Technical Report KSL-90-53. Stanford: University, Knowledge Systems Laboratory, 1990. URL: <http://ksl-web.stanford.edu/ KSL_Abstracts/KSL-90-53.html>. Consultado: 2002-12-08.

Gruber, T. R. (1991). The Role of Common Ontology in Achieving Sharable, Reusable Knowledge Bases. // Principles of Knowledge Representation and Reasoning: Proceedings of the Second International Conference. Cambridge: Morgan Kaufmann, 1991. 601-602. URL: <http://www-ksl.stanford.edu/knowledge-sharing/papers/role-ofonto.ps>. Consultado: 2002-12-08.

Gruber, T. R. (1992). Ontolingua: A mechanism to support portable ontologies: Technical Report KSL-91-66. Stanford: University, Knowledge Systems Laboratory, 1992. URL: <http://citeseer.nj.nec.com/cache/papers/cs/14913/ftp:zSzzSzksl.stanford.eduzSzpubzSzK SL_ReportszSz.zSzKSL-91-66.pdf/gruber92ontolingua.pdf>. Consultado: 2002-12-08.

Gruber, T. R. (1993a). A Translation Approach to Portable Ontology Specifications. // Knowledge Acquisition. 5: 2 (1993) 199-220. URL: <http://gicl.mcs.drexel.edu/ people/regli/Classes/KBA/Readings/KSL-92-71.pdf>. Consultado: 2002-12-08.

Gruber, T. R. (1993b). Toward principles for the design of ontologies used for knowledge sharing. // Guarino, N.; Poli, R. (eds.). Formal Ontology in Conceptual Analysis and Knowledge Representation: Stanford Knowledge Systems Laboratory Report KSL-9304. [s.1.]: Kluwer Academic. URL: <http://citeseer.nj.nec.com/cache/ papers/cs /490/http:zSzzSzwww-ks1. stanford.eduzSzknowledgesharingzSzpaperszSzonto-design.pdf/gruber93toward.pdf>. Consultado: 2002-12-08.

Gruber, T. R.; Olsen, G. R. (1994). An ontology for engineering mathematics. // Doyle, J.; Torasso, P.; Sandewall, E. (eds.). Fourth International Conference on Principles of Knowledge Representation and Reasoning. Bonn: Morgan Kaufmann, 1994. URL: <http://wwwksl.stanford.edu/knowledge-sharing/papers/engmath.html>. Consultado: 2002-12-08.

Gruber, T. R.; Tenenbaum, J. M.; Weber, J. C. (1992). Toward a knowledge medium for collaborative product development. // Proceedings of the Second International Conference on Artificial Intelligence in Design. [s.1.]: Kluwer Academic, 1992. 413-432. URL: <http://citeseer.nj.nec.com/cache/papers/cs/14917/ftp:zSzzSzksl.stanford.eduzSzpubzSzK SL_ReportszSz.zSzKSL-91-78.pdf/gruber92toward.pdf>. Consultado: 2002-12-08.

Guarino, N. (1993). The Role of Identity Conditions in Ontology Design. // Freksa, C.; Mark, D. M. (eds.). Spatial Information Theory: Cognitive and Computational Foundations of Geographic Information Science: Proceedings of International Conference COSIT '99. Berlin: Springer Verlag, 1999. 221-234. URL: <http://www.ladseb.pd.cnr.it/infor/ Ontology/Papers/IJCAI99.pdf $>$. Consultado: 2002-12-08.

Scire. $8: 1$ (en.-jun. 2002) 55-86. 
Guarino, N. (1994). The Ontological Level. // Casati, R.; Smith, B.; White, G. (eds.). Philosophy and the Cognitive Science. Vienna: Hölder-Pichler-Tempsky, 1994. 443-456. URL: <http://www.ladseb.pd.cnr.it/infor/Ontology/Papers/OntLev.pdf>. Consultado: 2002-12-08.

Guarino, N. (1995). Formal Ontology, Conceptual Analysis and Knowledge Representation. // International Journal of Human and Computer Studies. 43: 5/6 (1995) 625-640. URL: <http:// www.ladseb.pd.cnr.it/infor/Ontology/Papers/FormOntKR.pdf>. Consultado: 2002-12-08.

Guarino, N. (1996a). Il ruolo dell'ontologia formale per l'integrazione e la condivisione di conoscenze. // Proceedings of AI*IA-96: Atti della Giornata di Lavoro su "Accesso, estrazione e integrazione di conoscenza". Napoli: AI*IA, 1996. URL: <http://www.ladseb.pd.cnr.it/ infor/Ontology/ Papers/AIIA96.pdf>. Consultado: 2002-12-08.

Guarino, N. (1996b). Modellazione logica della conoscenza di prodotto: problemi e prospettive. // Proceedings of AI*IA 96: Atti della giornata di lavoro su "Intelligenza Artificiale per la gestione del know-how aziendale". Napoli: AI*IA, 1996. URL: <http:// www.ladseb.pd.cnr.it/infor/Ontology/Papers/AIIA96ind.pdf>. Consultado: 2002-12-08.

Guarino, N. (1997a). Understanding, Building, and Using Ontologies: A Commentary to "Using Explicit Ontologies in KBS Development", by van Heijst, Schreiber, and Wielinga. // International Journal of Human and Computer Studies. 46 (1997) 293-310. URL: <http://ontology.ip.rm.cnr.it/Papers/vanHeijst.pdf>. Consultado: 2002-12-08.

Guarino, N. (1997b). Semantic Matching: Formal Ontological Distinctions for Information Organization, Extraction, and Integration. // Pazienza, M. T. (ed.). Information Extraction: A Multidisciplinary Approach to an Emerging Information Technology. [s.1.]: Springer Verlag, 1997. 139-170. URL: <http://citeseer.nj.nec.com/cache/papers/cs/297/ http:zSzzSzwww.ladseb.pd.cnr.itzSzinforzSzOntologyzSzPaperszSzSCIE97.pdf/guarin 097semantic.pdf>. Consultado: 2002-12-08.

Guarino, N. (1997c). Some Organizing Principles for a Unified Top-Level Ontology: LADSEBCNR Internal Report 02/97. Padova: LADSEB, 1997. URL: <http://citeseer.nj.nec.com/cache/papers/cs/297/http:zSzzSzwww.ladseb.pd.cnr.itzSzinfor zSzOntologyzSzPaperszSzTopLevel.pdf/guarino97some.pdf>. Consultado: 2002-12-08.

Guarino, N. (1998a). Formal Ontology and Information Systems. // Guarino, N. (ed.) Formal Ontology in Information Systems: Proceedings of FOIS'98: Trento, Italy, 6-8 June 1998. Amsterdam: IOS Press, 1998. 3-15. URL: <http://www.ladseb.pd.cnr.it/infor/Ontology/ Papers/FOIS98.pdf>. Consultado: 2002-12-08.

Guarino, N. (1998b). Some Ontological Principles for Designing Upper Level Lexical Resources. // Rubio, A.; Gallardo, N.; Castro, R.; Tejada, A. Tejada (eds.). Proceedings of First International Conference on Language Resources and Evaluation. Granada: European Language Resources Association, 1998. 527-534. URL: <http://www.ladseb. pd.cnr.it/infor/Ontology/Papers/LREC98.pdf>. Consultado: 2002-12-08.

Guarino, N.; Borgo, S.; Massolo, C.(1997). Logical Modelling of Product Knowledge: Towards a Well-Founded Semantics for STEP. // Proceedings of European Conference on Product Data Technology (PDT Days 97). [s.1.: s.n.], 1997. 183-190. URL: <http:// www.ladseb.pd.cnr.it/infor/Ontology/Papers/PDT97.pdf>. Consultado: 2002-12-08.

Guarino, N.; Carrara, M.; Giaretta, P. (1993). An Ontology of Meta-Level Categories: LADSEB-CNR Int. Rep. 6/93: Preliminary version. Padova: LADSEB, 1993. URL: <http://citeseer.nj.nec.com/cache/papers/cs/3383/ftp:zSzzSzftp.cc.gatech.eduzSzpubzSz 
groupszSzaizSzsymposiazSzaaai-spring-95zSzpaperszSzrelatedpaperszSzontology.pdf/guarino94ontology.pdf>. Consultado: 2002-12-08.

Guarino, N.; Carrara, M.; Giaretta, P.(1994a). An Ontology of Meta-Level Categories. // Sandewall, E.; Torasso, P. (eds.). Principles of Knowledge Representation and Reasoning: Proceedings of the Fourth International Conference (KR94). San Mateo: Morgan Kaufmann, 1994. 270-280. URL: <http://citeseer.nj.nec.com/cache/papers/cs/3383/ftp:z SzzSzftp.cc. gatech.eduzSzpubzSzgroupszSzaizSzsymposiazSzaaai-spring-95zSzpa perszSzrelated-paperszSzontology.pdf/guarino94ontology.pdf >. Consultado: 2002-12-08.

Guarino, N.; Carrara, M.; Giaretta, P. (1994b). Formalizing Ontological Commitments. // Proceedings of National Conference on Artificial Intelligence (AAAI-94). Seattle: Morgan Kaufmann, 1994. 560-567. URL: <http://www.ladseb.pd.cnr.it/infor/Ontology/ Papers/ AAAI94.pdf>. Consultado: 2002-12-08.

Guarino, N.; Giaretta, P. (1995). Ontologies and Knowledge Bases: Towards a Terminological Clarification. // Mars, N. (ed.). Towards Very Large Knowledge Bases: Knowledge Building and Knowledge Sharing. Amsterdam: IOS Press, 1995. 25-32. URL: <http://ontology.ip.rm.cnr.it/Papers/KBKS95.pdf>. Consultado: 2002-12-08.

Guarino, N.; Massolo, C.; Vetere, G. (1999). OntoSeek: Content-Based Access to the Web. // IEEE Intelligent Systems. 14: 3 (1999) 70-80. URL: <http://citeseer.nj.nec.com/cache/ papers/cs/22433/http:zSzzSzwww.ladseb.pd.cnr.itzSzinforzSzOntologyzSzPaperszSzO ntoSeek.pdf/guarino99ontoseek.pdf>. Consultado: 2002-12-08.

Guarino, N.; Welty, C. (2000a). Ontological Analysis of Taxonomic Relationships. // Länder, A.; Storey, V. (eds.). Proceedings of ER-2000: The 19th International Conference on Conceptual Modeling. [s.1.]: Springer Verlag, 2000. URL: <http://www.cs.vassar.edu/ faculty/welty/papers/er2000/LADSEB05-2000.pdf>. Consultado: 2002-12-08.

Guarino, N.; Welty, C. (2000b). A Formal Ontology of Properties. // Dieng, R. Corby, O. (eds.). Knowledge Engineering and Knowledge Management: Methods, Models and Tools: 12th International Conference, EKAW2000. [s.1.]: Springer Verlag, 2000. 97-112. URL: <http://delicias.dia.fi.upm.es/WORKSHOP/ECAI00/12.pdf>. Consultado: 2002-12-08.

Guarino, N.; Welty, C. (2000c). Identity, Unity, and Individuality: Towards a Formal Toolkit for Ontological Analysis. // Werner, H. (ed.). ECAI-2000: The European Conference on Artificial Intelligence. Berlin: IOS Press, 2000. 219-223. URL: <http://www.ladseb. pd.cnr.it/infor/Ontology/Papers/LADSEB02-2000.pdf>. Consultado: 2002-12-08.

Guarino, N.; Welty, C. (2000d). Towards a methodology for ontology-based model engineering. // Proceedings of ECOOP-2000 Workshop on Model Engineering. [s.l.: s.n.], 2000. URL: <http://www.ladseb.pd.cnr.it/infor/Ontology/Papers/ecoop-00ws.pdf>. Consultado: 2002-12-08.

Guarino, N.; Welty, C. (2001). Identity and subsumption. // Green, R.; Bean C. A. Hyon Myaeng, S. (eds.). The Semantics of Relationships: An Interdisciplinary Perspective. [s.1.]: Kluwer, 2001. URL: <http://www.ladseb.pd.cnr.it/infor/Ontology/Papers/ Identity\&Subsumption.pdf >. Consultado: 2002-12-08.

Heflin, J.; Hendler, J.(2000a). Semantic Interoperability on the Web. // Proceedings of Extreme Markup Languages 2000. [s.1.]: Graphic Communications Association, 2000. 111-120. URL: 〈http://www.cs.umd.edu/projects/plus/SHOE/pubs/extreme2000.pdf>. Consultado: 2002-12-08.

Scire. $8: 1$ (en.-jun. 2002) 55-86. 
Heflin, J.; Hendler, J. (2000b). Dynamic Ontologies on the Web. // Proceedings of the Seventeenth National Conference on Artificial Intelligence (AAAI-2000). Menlo Park: AAAI/MIT Press, 2000. 443-449. URL: <http://www.cs.umd.edu/projects/plus/SHOE/ pubs/aaai2000.pdf>. Consultado: 2002-12-08.

Heflin, J.; Hendler, J. (2000c). Searching the Web with SHOE. // Artificial Intelligence for Web Search: Papers from the AAAI Workshop: WS-00-01. Menlo Park: AAAI Press, 2000. 35-40. URL: <http://citeseer.nj.nec.com/cache/papers/cs/15003/http:zSzz Szwww.cs.umd.eduzSzprojectszSzpluszSzSHOEzSzpubszSzaiweb2000.pdf/heflin00se arching.pdf>. Consultado: 2002-12-08.

Heflin, J.; Hendler, J.; Luke, S. (1998). Reading Between the Lines: Using SHOE to Discover Implicit Knowledge from the Web. // AI and Information Integration: Papers from the 1998 Workshop: WS-98-14. Menlo Park: AAAI Press, 1998. 51-57. URL: <http://citeseer.nj.nec.com/cache/papers/cs/660/http:zSzzSzwww.cs.umd.eduzSzproject szSzpluszSzSHOEzSzshoe-aaai98.pdf/heflin98reading.pdf>. Consultado: 2002-12-08.

Heflin, J.; Hendler, J.; Luke, S. (1999a). SHOE: A Knowledge Representation Language for Internet Applications: Technical Report CS-TR-4078 (UMIACS TR-99-71). Maryland: Deptartment of Computer Science, University of Maryland at College Park, 1999. URL: $<$ http://citeseer.nj.nec.com/cache/papers/cs/11142/ftp:zSzzSzftp.cs.umd.eduzSzpubzSz p a pers z S z p a pers z S z n c s tr 1. u m c p z S z C S - T R - 4078 z S z C S - T R 4078.pdf/heflin99shoe.pdf>. Consultado: 2002-12-08.

Heflin, J.; Hendler, J.; Luke, S. (1999b). Coping with Changing Ontologies in a Distributed Environment. // Ontology Management: Papers from the AAAI Workshop: WS-99-13. Menlo Park: AAAI Press, 1999. 74-79. URL: <http://www.cs.umd.edu/projects/plus/ SHOE/pubs/shoe-aaai99.pdf > Consultado: 2002-12-08.

Heflin, J.; Hendler, J.; Luke, S. (1999c). Applying Ontology to the Web: A Case Study. // Mira, J.; Sánchez-Andrés, J. (eds.). International Work-Conference on Artificial and Natural Neural Networks: IWANN'99: Proceedings. Berlin: Springer, 1999. II, 715-724. URL: <http://www.cs.umd.edu/projects/plus/SHOE/pubs/iwann99.pdf>. Consultado: 2002-12-08.

Hermjakob, U. (2001). Parsing and Question Classification for Question Answering. // Proceedings of the Workshop on Open-Domain Question Answering at the ACL-01 Conference. Toulouse: [s.n.], 2001. URL: <http://www.isi.edu/ ulf/papers/acl01-qaparsing.pdf>. Consultado: 2002-12-08.

Heylighen, F. (1990). Representation and Change: A Metarepresentational Framework for the Foundations of Physical and Cognitive Science. [s.1.]: Gent, 1990. URL: $<$ http://pespmc1.vub.ac.be/THESIS.html>. Consultado: 2002-12-08.

Hovy, E. H.; Gerber, L.; Hermjakob, U.; Junk, M.; Lin, C.-Y. (2000). Question Answering in Webclopedia. // Proceedings of the TREC-9 conference. Gaithersburg: NIST, 2000. URL: <http://rec.nist.gov/pubs/trec9/papers/webclopedia.pdf>. Consultado: 2002-12-08.

Hovy, E.H.; Gerber, L.; Hermjakob, U.; Lin, C.-Y.; Ravichandran, D. (2001). Toward Semantics-Based Answer Pinpointing. // Proceedings of the DARPA Human Language Technology conference (HLT). San Diego: [s.n.], 2001. URL: <http://www.isi.edu/ natural-language/projects/webclopedia/pubs/01hlt.pdf>. Consultado: 2002-12-08.

Hovy, E.H.; Philpot, A.; Ambite, J.-L.; Arens, Y.; Klavans, J.L.; Bourne, W.; Saroz, D. (2001). Data Acquisition and Integration in the DGRC's Energy Data Collection 
Project. // Proceedings of the Digital Government 2001 Conference. Los Angeles: [s.n.], 2001. URL: <http://www.isi.edu/naturallanguage/people/hovy/papers/01dg.o.pdf>. Consultado: 2002-12-08.

Karp, P. D.; Gruber, T. R. (1994). A Generic Knowledge-Base Access Protocol: International Technical Report. [s.1.]: SRI, 1994. URL: <http://ksl-web.stanford.edu/knowledgesharing/papers/index.html\#gfp>. Consultado: 2002-12-08.

Klein, M. C. A. (2000). The Relation between Ontologies and Schema-Languages: Translating OIL-Specifications to XML-Schema. // Proceedings of the Workshop on Applications of Ontologies and Problem-solving Methods: 14th European Conference on Artificial Intelligence: ECAI-00. Berlin: [s.n.], 2000. URL: <http://www.cs.vu.nl/ mcaklein/ papers/oil-xmls.pdf>. Consultado: 2002-12-08.

Knight, K. (1996). Learning Word Meanings by Instruction. // Proceedings of the American Association of Artificial Intelligence: AAAI-96. Menlo Park: AAAI Press, 1996. URL: <http://citeseer.nj.nec.com/cache/papers/cs/385/http:zSzzSzwww.isi.eduzSznaturallanguagezSzmtzSzinv-unify.pdf/knight96learning.pdf >. Consultado: 2002-12-08.

Knight, K. (1997). Automating Knowledge Acquisition for Machine Translation. // AI Magazine. 18: 4 (1997). URL: <http://citeseer.nj.nec.com/cache/papers/cs/385/http:zSzzSzwww.isi. eduzSznatural-languagezSzmtzSzaimag97.pdf/knight97automating.pdf >. Consultado: 200212-08.

Knight, K. (1999). Decoding Complexity in Word-Replacement Translation Models. // Computational Linguistics. 25: 4 (1999). URL: <http://acl.ldc.upenn.edu/J/J99/J994005.pdf>. Consultado: 2002-12-08.

Knight, K.; Chander, I. (1994). Automated Postediting of Documents. // Proceedings of the American Association of Artificial Intelligence AAAI-94. Menlo Park: AAAI Press, 1994. URL: <http://arxiv.org/PS_cache/cmp-lg/pdf/9407/9407028.pdf>. Consultado: 2002-12-08.

Knight, K.; Chander, I.; Haines, M.; Hatzivassiloglou, V.; Hovy, E.H.; Iida, M.; Luk, S.K.; Okumura, A.; Whitney, R.A.; Yamada, K. (1994). Integrating Knowledge Bases and Statistics in MT.// Proceedings of the 1st AMTA Conference. Columbia: [s.n.], 1994. URL: <http://www.isi.edu/natural-language/people/knight.html>. Consultado: 2002-12-08.

Knight, K.; Chander, I.; Haines, M.; Hatzivassiloglou, V.; Hovy, E.H.; Iida, M. Luk, S.K.; Whiteny, R.A.; Yamada, K. (1995). Filling Knowledge Gaps in a Broad-Coverage MT System. // Proceedings of the 14th IJCAI Conference. Montreal, Quebéc: [s.n.], 1995. URL: <http://arxiv.org/PS_cache/cmp-lg/pdf/9506/9506009.pdf>. Consultado: 2002-12-08.

Knight, K.; Graehl, J. (1997). Machine Transliteration. // Proceedings of the ACL-97. Madrid: [s.n.], 1997. URL: <http://citeseer.nj.nec.com/cache/papers/cs/385/http:zSzzSzwww.isi. eduzSznatural-languagezSzmtzSztransliterate.pdf/knight97machine.pdf $>$. Consultado: 2002-12-08.

Knight, K.; Hatzivassiloglou, V. (1995). Two-Level, Many-Paths Generation. // Proceedings of the ACL-95. Cambridge: [s.n.], 1995. URL: <http://acl.ldc.upenn.edu/P/P95/P951034.pdf>. Consultado: 2002-12-08.

Knight, K.; Luk, S. (1994). Building a Large-Scale Knowledge Base for Machine Translation. // Proceedings of the American Association of Artificial Intelligence AAAI-94. Menlo Park: AAAI Press, 1994. URL: <http://arxiv.org/PS_cache/cmp-1g/pdf/ 9407/9407029.pdf>. Consultado: 2002-12-08.

Scire. $8: 1$ (en.-jun. 2002) 55-86. 
Knight, K.; Yamada, K. A (1999). Computational Approach to Deciphering Unknown Scripts. // Proceedings of the ACL Workshop on Unsupervised Learning in Natural Language Processing. [s.1.: s.n.], 1999. URL: <http://www.isi.edu/natural-language/ mt/decipher.ps>. Consultado: 2002-12-08.

Koehn, P.; Knight, K. (2001). Knowledge Sources for Word-Level Translation Models. // Empirical Methods in Natural Language Processing conference (EMNLP'01). [s.1.: s.n.], 2001. URL: 〈http://www.isi.edu/ koehn/publications/emnlp2001.pdf>. Consultado: 2002-12-08.

Langkilde, I.; Knight, K. (1998a). The Practical Value of N-Grams in Generation. // Proceedings of the International Natural Language Generation Workshop. Niagra-on-theLake, Ontario: [s.n.], 1998. URL: 〈http://www.isi.edu/licensed-sw/halogen/genprac.ps〉. Consultado: 2002-12-08.

Langkilde, I.; Knight, K. (1998b). Generation that Exploits Corpus-based Statistical Knowledge. // Proceedings of the ACL/COLING-98. Montreal, Quebéc: [s.n.], 1998. URL: <http://citeseer.nj.nec.com/cache/papers/cs/385/http:zSzzSzwwwscf.usc.eduzSz langkildzSzpaperszSznitro98.pdf/langkilde98generation.pdf >. Consultado: 2002-12-08.

Luke, S.; Hendler, J. (1997). Web Agents That Work. // IEEE Multimedia. 4: 3 (JulySeptember 1997). URL: 〈http://www.cs.umd.edu/users/seanl/papers/multimedia.pdf>. Consultado: 2002-12-08.

Luke, S. (1998). A Revised Comparison of Crossover and Mutation in Genetic Programming. // Genetic Programming (GP98) conference proceedings. Madison: [s.n.], 1998. URL: <http://citeseer.nj.nec.com/cache/papers/cs/660/http:zSzzSzwww.cs.umd.eduzSzusersz SzseanlzSzpaperszSzrevisedgp98.pdf/luke98revised.pdf>. Consultado: 2002-12-08.

Luke, S.; Spector, L.; Rager, D. (1996a). Ontology-Based Knowledge Discovery on the World-Wide Web. // AAAI96 Workshop on Internet-based Information Systems. Menlo Park: AAAI Press, 1996. URL: <http://www.cs.umd.edu/projects/plus/SHOE/pubs/aaaipaper.html>. Consultado: 2002-12-08.

Luke, S.; Spector, L. (1996b). Evolving Graphs and Networks with Edge Encoding: Preliminary Report. // Late-breaking Papers of the Genetic Programming (GP96) Conference. Stanford: [s.n.], 1996. URL: <http://citeseer.nj.nec.com/cache/papers/cs/140/http:zSzzSzwww.cs.umd. eduzSz $\sim$ seanlzSzpaperszSzgraph-paper.pdf/luke96evolving.pdf>. Consultado: 2002-12-08.

Luke, S.; Spector, L.; Rager, D.; Hendler, J. (1997). Ontology-based Web Agents. // Proceedings of First International Conference on Autonomous Agents: AA-97. [s.l.: s.n.], 1997. URL: <http:// citeseer.nj.nec.com/cache/papers/cs/2124/http:zSzzSzgopher.cs.umbc.eduzSzcourseszSzgra duatezSz891zSzcurrentzSzshoe.pdf/luke97ontologybased.pdf>. Consultado: 2002-12-08.

Marcu, D. (2001). Towards a Unified Approach to Memory- and Statistical-Based Machine Translation. Proceedings of ACL-01. Toulouse: [s.n.], 2001. URL: <http://www.isi.edu/ marcu/papers/transmem-ac101.pdf>. Consultado: 2002-12-08.

Marcu, D.; Carlson, L.; Watanabe, M. (2002). The Automatic Translation of Discourse Structures. // Association for Computational Linguistics conference, North American chapter (NAACL'00). [s.1.: s.n.], 2000. URL: <http://acl.ldc.upenn.edu/A/A00/A002002.pdf>. Consultado: 2002-12-08.

McGuire, J. G.; Kuokka, D. R.; Weber, J. C.; Tenenbaum, J. M.; Gruber, T. R.; Olsen, G. R. (1993). SHADE: Technology for Knowledge-Based Collaborative Engineering. // 
Journal of Concurrent Engineering: Applications and Research (CERA). 1: 2 (September 1993). URL: <http://citeseer.nj.nec.com/cache/papers/cs/3117/ftp: zSzzSzksl.stanford.eduz SzpubzSzknowledge-sharingzSzpaperszSzshadecera.pdf/mcguire93shade.pdf $>$. Consultado: 2002-12-08.

Musen, M. A. (1992). Dimensions of knowledge sharing and reuse. // Computers and Biomedical Research. 25 (1992). 435-467. URL: <http://www-ksl.stanford.edu/ knowledge-sharing/papers/\#musen-dimensions>. Consultado: 2002-12-08.

Musliner, D. (1993). CIRCA: A Cooperative Intelligent Real-Time Control Architecture. // IEEE Transactions on Systems, Man, and Cybernetics. 23: 6 (1993). URL: <http://citeseer.nj.nec.com/cache/papers/cs/986/http:zSzzSzftp.eecs.umich.eduzSzpeopl ezSzdurfeezSzsmc93.pdf/musliner93circa.pdf>. Consultado: 2002-12-08.

Olsen, G. R.; Cutkosky, M.; Tenenbaum, J. M.; Gruber, T. R.(1994). Collaborative Engineering based on Knowledge Sharing Agreements. // Proceedings of the 1994 ASME Database Symposium. Minneapolis: [s.n.], 1994. URL: <http://citeseer.nj.nec.com/cache/papers/cs/17509/ftp:zSzzSzwwwcdr.stanford.eduzSzpubzSzCDRzSzPublicationszSzReportszSzCollabEngr_ASMEData base94.pdf/olsen94collaborative.pdf>. Consultado: 2002-12-08.

Patil, R. S.; Fikes, R. E.; Patel-Schneider, P. F.; McKay, D.; Finin, T.; Gruber, T. R.; Neches, R. (1992). The DARPA Knowledge Sharing Effort: Progress report. // Rich, C.; Nebel, B.; Swartout, W. (eds.). Principles of Knowledge Representation and Reasoning: Proceedings of the Third International Conference. Cambridge: Morgan Kaufmann, 1992. URL: <http://citeseer.nj.nec.com/cache/papers/cs/14917/ftp:zSzzSzksl. stanford.eduzSzpubzSzKSL_ReportszSz.zSzKSL-93-23.pdf/pati193darpa.pdf>. Consultado: 2002-12-08.

Spector, L.; Luke, S. (1996a). Cultural Transmission of Information in Genetic Programming. // Genetic Programming (GP96) conference proceedings. Cambridge: The MIT Press, 1996. URL: <http://citeseer.nj.nec.com/cache/papers/cs/140/http:zSzzSzhamp. hampshire.eduzSz lasCCSzSzpubszSzculture-gp96-e.pdf/spector96cultural.pdf〉. Consultado: 2002-12-08.

Spector, L.; Luke, S. (1996b). Culture Enhances the Evolvability of Cognition. // Cognitive Science (CogSci96) conference proceedings. [s.1.: s.n.], 1996. URL: <http://citeseer.nj.nec.com/cache/papers/cs/140/http:zSzzSzhamp.hampshire.eduzSz la sCCSzSzpubszSzculture-cogsci-e.pdf/spector96culture.pdf >. Consultado: 2002-12-08.

Stoffel, K.; Taylor, M.; Hendler, J. (1997). Efficient Management of Very Large Ontologies. // Proceedings of American Association for Artificial Intelligence Conference (AAAI-97). Menlo Park: AAAI/MIT Press, 1997. URL: <http://citeseer.nj.nec.com/cache/papers/ cs/660/http:zSzzSzwww.cs.umd.eduzSzprojectszSzpluszSzParkazSzaaai97.pdf/stoffe19 7efficient.pdf >. Consultado: 2002-12-08.

Stuckenschmidt, H. (2000). Using OIL for Intelligent Information Integration // Proceedings of the Workshop on Applications of Ontologies and Problem-Solving Methods at ECAI 2000. [s.1.: s.n.], 2000. URL: <http://www.informatik.unibremen.de/ heiner/public/ECAI-00ws.pdf>. Consultado: 2002-12-08.

Stuckenschmidt, H.; Wache, H.(2000). Context Modeling and Transformation for Semantic Interoperability // Knowledge Representation meets Databases: Proceedings of the

Scire. $8: 1$ (en.-jun. 2002) 55-86. 
Workshop at ECAI 2000. [s.1.: s.n.], 2000. URL: <http://citeseer.nj.nec.com/ cache/papers/cs/16838/http:zSzzSzwww.tzi.orgzSz wachezSzPaperszSzkrdb-00wache.pdf/stuckenschmidt00context.pdf>. Consultado: 2002-12-08.

Taylor, M.; Stoffel, K.; Hendler, J. (1997). Ontology-based Induction of High Level Classification Rules. // SIGMOD Data Mining and Knowledge Discovery workshop proceedings. Tuscon: [s.n.], 1997. URL: <http://citeseer.nj.nec.com /cache/papers/cs/11353/http:zSzzSzwww.cs.umd.eduzSz mtaylorzSzsigmoddmkd97 .pdf/ontology-based-induction-of.pdf>. Consultado: 2002-12-08.

Taylor, M.; Stoffel, K.; Hendler, J.; Saltz, J. (1998). Using Distributed Query Result Caching to Evaluate Queries for Parallel Data Mining Algorithms. // Proceedings of the PTPTA98 Conference on Data Warehousing. Las Vegas: [s.n.], 1998. URL: <http://www.cs.umd.edu/users/mtaylor/pdpta98.ps>. Consultado: 2002-12-08.

Tsuneto, R.; Hendler, J.; Nau, D.; Barros, L. De. (1998). Matching Problem Features with Task Selection for Better Performance in HTN Planning. // AIPS Workshop on Knowledge Engineering and Acquisition for Planning. [s.1.: s.n.], 1998. URL: <http://www.cs.umd.edu/users/reiko/>. Consultado: 2002-12-08.

Tsuneto, R.; Nau, D.; Hendler, J. (1997). Plan-Refinement Strategies and Search-Space Size. // Proceedings of the Fourth European Conferencfe on Planning (ECP-97). [s.1.: s.n.], 1997. URL: <http://www.cs.umd.edu/users/reiko/>. Consultado: 2002-12-08.

Tsuneto, R.; Erol, K.; Hendler, J.; Nau, D. (1996). Commitment Strategies in Hierarchical Task Network Planning. // AAAI-96. Menlo Park: AAAI Press, 1996. URL: <http://www.cs.umd.edu/users/reiko/>. Consultado: 2002-12-08.

Walther, E.; Eriksson, H.; Musen, M. A. (1992). Plug and play: Construction of task-specific expert-system shells using sharable context ontologies: Technical Report KS1-92-40. Stanford : Knowledge Systems Laboratory, University, 1992. URL: <http://citeseer.nj. nec.com/cache/papers/cs/26268/http:zSzzSzwww-smi.stanford.eduzSzpubszSzSMI_ ReportszSzSMI-92-0437.pdf/walther92plugplay. pdf>. Consultado: 2002-12-08.

Welty, C.; Guarino, N. (2001). Supporting Ontological Analysis of Taxonomic Relationships. // Data and Knowledge Engineering. (September 2001). URL: <http://www. ladseb.pd.cnr.it/ infor/Ontology/Papers/TR-08-2001.pdf>. Consultado: 2002-12-08.

Yamada, K. A. (1996). Controlled Skip Parser. // Proceedings of the 2nd AMTA Conference. Montreal, Quebéc: [s.n.], 1996. URL: 〈http://www.isi.edu/natural-language/projects/ GAZELLE.html>. Consultado: 2002-12-08.

Yamada, K.; Knight, K. A. (2001). Syntax-Based Statistical Translation Model. // Proceedings of ACL-01. Toulouse: [s.n.], 2001. URL: <http://citeseer.nj.nec.com/cache/papers/cs/ 22655/http:zSzzSzwww.isi.eduzSznatural-languagezSzprojectszSzrewritez Szsyntax.pdf/a-syntax-based-statistical.pdf>. Consultado: 2002-12-08. 